RIMS-953

December 1993

\title{
Two-Dimensional Gravity and Nonlinear Gauge Theory
}

\author{
NORIAKI IKEDA \\ Research Institute for Mathematical Sciences \\ Kyoto University, Kyoto 606-01, Japan
}

\begin{abstract}
We construct a gauge theory based on nonlinear Lie algebras, which is an extension of the usual gauge theory based on Lie algebras. It is a new approach to generalization of the gauge theory. The two-dimensional gravity is derived from nonlinear Poincaré algebra, which is the new Yang-Mills like formulation of the gravitational theory. As typical examples, we investigate $R^{2}$ gravity with dynamical torsion and generic form of 'dilaton' gravity. The supersymmetric extension of this theory is also discussed.
\end{abstract}




\section{Introduction}

The gauge theory is fundamental object in quantum field theory. In this paper, we consider a generalization of the usual gauge theory based on Lie algebra and its application.

There are many studies to extend it by modifying symmetry structure, such as supersymmetry, ${ }^{[1]}$ quantum group, ${ }^{[2]} \mathrm{W}$ algebra. ${ }^{[3]}$ However in this paper, we consider quite new approach to extend the usual gauge theory. We construct a twodimensional gauge theory based on nonlinear extension of Lie algebras as a generalization of the usual nonabelian gauge theory with internal gauge symmetry. We call it nonlinear gauge theory, which is a new generalization of nonabelian gauge theory.

Nonlinear extension of Lie algebras which is called nonlinear Lie algebra has the following commutation relation:

$$
\left[T_{A}, T_{B}\right]=W_{A B}(T)
$$

where $W_{A B}(T)$ denotes a polynomial in $T_{C}$. The details of definition are presented in section 2.2. Quadratic nonlinear Lie algebras in the context of quantum field theory were first introduced by K. Schoutens, A. Sevrin and P. van Nieuwenhuizen. ${ }^{[6]}$ They mainly analyzed the W-gravity as an application of nonlinear Lie algebra to quantum field theory. Our nonlinear gauge theory is different realization of nonlinear Lie algebra in quantum field theory, and presents a new category of constrainted systems.

The two-dimensional nonlinear gauge theory is closely related to two-dimensional gravity. The investigation of diffeomorphism-invariant field theories is expected to provide indispensable information for the quest of quantum gravity. At the classical level, the Einstein theory - field theory of metric tensor with general covariance - is quite successful in describing gravitational phenomena. 
The general covariance in gravitation theory is external gauge symmetry, which is spacetime symmetry. It may be called gauge symmetry of the Utiyama type. ${ }^{[4]}$ On the other hand, the internal symmetry such as in QED or QCD is called the one of the Yang-Mills type. ${ }^{[5]}$ The connection to gauge symmetry of the Utiyama type with the Yang-Mills type has been investigated on various authors. Witten's three-dimensional Einstein gravity (Chern-Simons-Witten gravity) can be regarded as one example for the above description. ${ }^{[7]}$ In two dimension, Jackiw-Teitelboim's model $^{[8]}$ is a typical example that a gravitational theory and a gauge theory have classically the same action. ${ }^{[9]}$ One of the reasons for investigating the connection between two types of symmetries is that symmetry of the Yang-Mills type is easier to treat in quantization problems than that of the Utiyama type. In this paper, we introduce new examples which have such properties and are related to nonlinear gauge theory.

The particular feature of two-dimensional nonlinear gauge theory is that it includes two-dimensional gravitation theory. When the nonlinear algebra is Lorentzcovariant extension of the Poincaré algebra, the theory turns out to be the YangMills-like formulation of $R^{2}$ gravity with dynamical torsion, ${ }^{[10][11]}$ or generic form of 'dilaton' gravity. ${ }^{[12]}$ The hidden symmetry of their gravitation theory is clarified. It yields new examples of the connection between the two types of gauge symmetries. Moreover a gauge theory of nonlinear Lie superalgebra is related to supergravity. When nonlinear superalgebra is nonlinear and Lorentz-covariant extension of super-Poincaré algebra, the theory turn out to be dilaton supergravity. ${ }^{[13]}$

The remaining part of the paper is organized as follows: In chapter 2, our action for 'nonlinear' gauge theory is constructed in quite a general manner. We also consider nonlinear Lie algebras as a background structure for the 'nonlinear' gauge theory. The BRS formulation of nonlinear gauge theory is formulated. The chapter 3 provides Lorentz-covariant quadratic extension of the Poincaré algebra and our nonlinear gauge theory based on it. It turn out that the resulting theory is the Yang-Mills-like formulation of $R^{2}$ gravity with dynamical torsion. In chapter 4 , the relevance of this 'nonlinear' gauge theory to the 'dilaton' gravity is clarified. 
In chapter 5, our action for 'nonlinear' gauge theory is extended to the supergauge theory. The connection to the dilaton supergravity of 'nonlinear' supergauge theory is analyzed. Chapter 6 concludes the paper.

\section{2. 'Nonlinear' Gauge Theory}

In this chapter, we explain a generic approach to the construction of a 'nonlinear' gauge theory - gauge theory based on nonlinear extension of Lie algebras. ${ }^{[12]}$

\section{1. 'Nonlinear' Gauge Transformation}

We introduce a 'coadjoint' scalar field $\Phi_{A}$ in addition to a vector field $h_{\mu}^{A}$ for the purpose of constructing a 'nonlinear' gauge theory. Here $A$ denotes an internal index. We consider the following extension of gauge transformation:

$$
\begin{aligned}
\delta(c) h_{\mu}^{A} & =\partial_{\mu} c^{A}+U_{B C}^{A}(\Phi) h_{\mu}^{B} c^{C}, \\
\delta(c) \Phi_{A} & =W_{B A}(\Phi) c^{B},
\end{aligned}
$$

where $\delta(c)$ is a gauge transformation with the gauge-transformation parameter $c^{A}$, and $U_{B C}^{A}(\Phi)$ and $W_{A B}(\Phi)$ are smooth functions of the field $\Phi_{A}$ to be determined below. In the case of usual nonabelian gauge theory, $U_{B C}^{A}=f_{B C}^{A}$ and $W_{B A}=$ $f_{B A}^{C} \Phi_{C}$, where $f_{A B}^{C}$ denote structure constants in some Lie algebra. Since the above gauge transformation should reduce to the usual nonabelian one if $U_{B C}^{A}$ is constant and $W_{B A}$ is a linear function of $\Phi_{C}$, we assume $U_{B C}^{A}=-U_{C B}^{A}$ and $W_{B A}=-W_{A B}$.

We investigate the commutator algebra of these gauge transformations with gauge-transformation parameters $c_{1}$ and $c_{2}$. We require a closed algebra on the scalar field $\Phi_{A}$

$$
\left[\delta\left(c_{1}\right), \delta\left(c_{2}\right)\right] \Phi_{A}=\delta\left(c_{3}^{\prime}\right) \Phi_{A},
$$

First we consider the case that the gauge parameters $c_{1}$ and $c_{2}$ are independent of 
$\Phi_{A}$. In this case, the right-hand side of (2.2) becomes

$$
\left[\delta\left(c_{1}\right), \delta\left(c_{2}\right)\right] \Phi_{A}=\left(\frac{\partial W_{C A}}{\partial \Phi_{D}} W_{B D}+\frac{\partial W_{A B}}{\partial \Phi_{D}} W_{C D}\right) c_{1}^{B} c_{2}^{C} .
$$

In case of nonabelian gauge theory, since $W_{B A}=f_{B A}^{C} \Phi_{C}$, we can rewrite (2.3) as

$$
\begin{aligned}
{\left[\delta\left(c_{1}\right), \delta\left(c_{2}\right)\right] \Phi_{A} } & =\left(f_{C A}^{D} f_{B D}^{E}+f_{A B}^{D} f_{C D}^{E}\right) \Phi_{E} c_{1}^{B} c_{2}^{C}, \\
& =f_{B C}^{D} f_{D A}^{E} \Phi_{E} c_{1}^{B} c_{2}^{C}, \\
& =\delta\left(c_{3}^{\prime}\right) \Phi_{A},
\end{aligned}
$$

by using the Jacobi identity of Lie algebra:

$$
f_{[A B}^{D} f_{C] D}^{E}=0
$$

where $[A B C]$ denotes antisymmetrization in the indices $A, B$, and $C$, and

$$
c_{3}^{A}=f_{B C}^{A} c_{1}^{B} c_{2}^{C} .
$$

In order to extend the above construction to (2.3), we replace the structure constant $f_{B A}^{C}$ by $\frac{\partial W_{B A}}{\partial \Phi_{C}}$ and $f_{B A}^{C} \Phi_{C}$ by $W_{B A}$ in (2.4). Then (2.4) becomes (2.3), and (2.5) is generalized to

$$
\frac{\partial W_{[A B}}{\partial \Phi_{D}} W_{C] D}=0 .
$$

Owing to this generalized Jacobi identity, (2.3) becomes

$$
\begin{aligned}
{\left[\delta\left(c_{1}\right), \delta\left(c_{2}\right)\right] \Phi_{A} } & =\left(\frac{\partial W_{C A}}{\partial \Phi_{D}} W_{B D}+\frac{\partial W_{A B}}{\partial \Phi_{D}} W_{C D}\right) c_{1}^{B} c_{2}^{C} \\
& =W_{D A} \frac{\partial W_{B C}}{\partial \Phi_{D}} c_{1}^{B} c_{2}^{C} .
\end{aligned}
$$

This assumption results in the composite parameter

$$
c_{3}^{\prime A}=\frac{\partial W_{B C}}{\partial \Phi_{A}} c_{1}^{B} c_{2}^{C},
$$

and the gauge algebra becomes the required form (2.2) on $\Phi_{A}$. In the next section, we show that the expression (2.6) can be derived as the Jacobi identity for a nonlinear Lie algebra with structure functions $W_{A B}$. 
Since the right-hand side of (2.8) involves $\Phi_{A}$, we have to consider the case that the gauge parameters $c_{4}$ and $c_{5}$ are dependent on $\Phi_{A}$ in $\left[\delta\left(c_{4}\right), \delta\left(c_{5}\right)\right] \Phi_{A}$. In this case, we have

$$
\begin{aligned}
{\left[\delta\left(c_{4}\right), \delta\left(c_{5}\right)\right] \Phi_{A}=} & \left(\frac{\partial W_{C A}}{\partial \Phi_{D}} W_{B D}+\frac{\partial W_{A B}}{\partial \Phi_{D}} W_{C D}\right) c_{4}^{B} c_{5}^{C} \\
& -\left[W_{D A} W_{B C}\left(\frac{\partial c_{5}^{D}}{\partial \Phi_{B}} c_{4}^{C}-\frac{\partial c_{4}^{D}}{\partial \Phi_{B}} c_{5}^{C}\right)\right] \\
= & W_{D A}\left[\frac{\partial W_{B C}}{\partial \Phi_{D}} c_{4}^{B} c_{5}^{C}-W_{B C}\left(\frac{\partial c_{5}^{D}}{\partial \Phi_{B}} c_{4}^{C}-\frac{\partial c_{4}^{D}}{\partial \Phi_{B}} c_{5}^{C}\right)\right]
\end{aligned}
$$

by using (2.6), and if we set

$$
c_{6}^{\prime A}=\frac{\partial W_{B C}}{\partial \Phi_{A}} c_{4}^{B} c_{5}^{C}-W_{B C}\left(\frac{\partial c_{5}^{A}}{\partial \Phi_{B}} c_{4}^{C}-\frac{\partial c_{4}^{A}}{\partial \Phi_{B}} c_{5}^{C}\right),
$$

the gauge algebra satisfies

$$
\left[\delta\left(c_{4}\right), \delta\left(c_{5}\right)\right] \Phi_{A}=\delta\left(c_{6}^{\prime}\right) \Phi_{A}
$$

On the other hand, as the commutator of two transformations on the vector field $h_{\mu}^{A}$, we require

$$
\left[\delta\left(c_{1}\right), \delta\left(c_{2}\right)\right] h_{\mu}^{A}=\delta\left(c_{3}\right) h_{\mu}^{A}+\cdots,
$$

where the ellipsis indicates a term which is irrelevant here. If we calculate the right-hand side of (2.11) explicitly, it is obtained that

$$
\begin{aligned}
{\left[\delta\left(c_{1}\right), \delta\left(c_{2}\right)\right] h_{\mu}^{A}=} & \partial_{\mu}\left(U_{B C}^{A} c_{1}^{B} c_{2}^{C}\right)-\frac{\partial U_{B C}^{A}}{\partial \Phi_{D}} \partial_{\mu} \Phi_{D} \\
& +\left(U_{D C}^{A} U_{B E}^{D}+U_{D E}^{A} U_{C B}^{D}+\frac{\partial U_{B C}^{A}}{\partial \Phi_{D}} W_{E D}+\frac{\partial U_{E B}^{A}}{\partial \Phi_{D}} W_{C D}\right) h_{\mu}^{B} c_{1}^{E} c_{2}^{C}
\end{aligned}
$$

where we assume that $c_{1}$ and $c_{2}$ are independent of $\Phi_{A}$. From the first term of 
left-hand side of (2.12), the composite parameter $c_{3}$ should be

$$
c_{3}^{A}=U_{B C}^{A} c_{1}^{B} c_{2}^{C}
$$

We require $c_{3}=c_{3}^{\prime}$, that is, we henceforth put

$$
U_{B C}^{A}(\Phi)=\frac{\partial W_{B C}}{\partial \Phi_{A}}
$$

in order for the commutator algebra to represent a consistent composition law of Lagrangian symmetry. Then (2.12) becomes

$$
\begin{aligned}
{\left[\delta\left(c_{1}\right), \delta\left(c_{2}\right)\right] h_{\mu}^{A} } & =\partial_{\mu}\left(\frac{\partial W_{B C}}{\partial \Phi_{A}} c_{1}^{B} c_{2}^{C}\right)-\frac{\partial^{2} W_{B C}}{\partial \Phi_{A} \partial \Phi_{D}} \partial_{\mu} \Phi_{D} \\
+\left(\frac{\partial W_{D C}}{\partial \Phi_{A}}\right. & \left.\frac{\partial W_{B E}}{\partial \Phi_{D}}+\frac{\partial W_{D E}}{\partial \Phi_{A}} \frac{\partial W_{C B}}{\partial \Phi_{D}}+\frac{\partial^{2} W_{B C}}{\partial \Phi_{A} \partial \Phi_{D}} W_{E D}+\frac{\partial^{2} W_{E B}}{\partial \Phi_{A} \partial \Phi_{D}} W_{C D}\right) h_{\mu}{ }^{B} c_{1}^{E} c_{2}^{C} \\
& =\partial_{\mu}\left(\frac{\partial W_{B C}}{\partial \Phi_{A}} c_{1}^{B} c_{2}^{C}\right)+\frac{\partial W_{B D}}{\partial \Phi_{A}} h_{\mu}{ }^{B} \frac{\partial W_{E C}}{\partial \Phi_{D}} c_{1}^{E} c_{2}^{C}-c_{1}^{C} c_{2}^{D} \frac{\partial^{2} W_{C D}}{\partial \Phi_{A} \partial \Phi_{B}} D_{\mu} \Phi_{B} \\
& =\delta\left(c_{3}\right) h_{\mu}^{A}-c_{1}^{C} c_{2}^{D} \frac{\partial^{2} W_{C D}}{\partial \Phi_{A} \partial \Phi_{B}} D_{\mu} \Phi_{B}
\end{aligned}
$$

by using (2.6).

In the case that $c_{4}$ and $c_{5}$ is dependent on $\Phi_{A}$, the commutator of two transformations on $h_{\mu}{ }^{A}$ is calculated by using (2.6) as follows:

$$
\left[\delta\left(c_{4}\right), \delta\left(c_{5}\right)\right] h_{\mu}^{A}=\delta\left(c_{6}\right) h_{\mu}^{A}-c_{4}^{C} c_{5}^{D} \frac{\partial^{2} W_{C D}}{\partial \Phi_{A} \partial \Phi_{B}} D_{\mu} \Phi_{B},
$$

where

$$
c_{6}^{A}=\frac{\partial W_{B C}}{\partial \Phi_{A}} c_{4}^{B} c_{5}^{C}+W_{B C}\left(\frac{\partial c_{5}^{D}}{\partial \Phi_{B}} c_{4}^{C}-\frac{\partial c_{4}^{D}}{\partial \Phi_{B}} c_{5}^{C}\right),
$$

which is the same as (2.10) and gives the consistent gauge algebra. 
The resulting form of the gauge transformation is given by

$$
\begin{aligned}
& \delta(c) h_{\mu}^{A}=\partial_{\mu} c^{A}+\frac{\partial W_{B C}(\Phi)}{\partial \Phi_{A}} h_{\mu}^{B} c^{C}, \\
& \delta(c) \Phi_{A}=W_{B A}(\Phi) c^{B}
\end{aligned}
$$

in terms of the functions $W_{A B}$ which satisfy the condition (2.6). This gauge transformation reduces to the usual nonabelian one when $W_{B A}(\Phi)=f_{B A}^{C} \Phi_{C}$.

Then the gauge algebra is given by

$$
\begin{aligned}
& {\left[\delta\left(c_{1}\right), \delta\left(c_{2}\right)\right] h_{\mu}^{A}=\delta\left(c_{3}\right) h_{\mu}^{A}-c_{1}^{C} c_{2}^{D} \frac{\partial^{2} W_{C D}}{\partial \Phi_{A} \partial \Phi_{B}} D_{\mu} \Phi_{B}} \\
& {\left[\delta\left(c_{1}\right), \delta\left(c_{2}\right)\right] \Phi_{A}=\delta\left(c_{3}\right) \Phi_{A}}
\end{aligned}
$$

where we have defined

$$
D_{\mu} \Phi_{A}=\partial_{\mu} \Phi_{A}+W_{A B}(\Phi) h_{\mu}^{B}
$$

We see the gauge transform of (2.18):

$$
\delta(c)\left(D_{\mu} \Phi_{A}\right)=\left(D_{\mu} \Phi_{C}\right) \frac{\partial W_{B A}}{\partial \Phi_{C}} c^{B} .
$$

This reveals that the object $D_{\mu}$ may be recognized as a covariant differentiation, $D_{\mu} \Phi_{A}$ transforming as a coadjoint vector. If the algebra is nondegenerate, it turns out that the gauge algebra is open as seen from (2.17) except for the case $\frac{\partial^{2} W_{C D}}{\partial \Phi_{A} \partial \Phi_{B}}=$ 0 , that is, the case of nonabelian gauge transformation.

The commutator of two covariant differentiations provides

$$
\left[D_{\mu}, D_{\nu}\right] \Phi_{A}=W_{A B}(\Phi) R_{\mu \nu}^{B}
$$

where the curvature $R_{\mu \nu}^{A}$ is defined by

$$
R_{\mu \nu}^{A}=\partial_{\mu} h_{\nu}^{A}-\partial_{\nu} h_{\mu}^{A}+\frac{\partial W_{B C}}{\partial \Phi_{A}} h_{\mu}^{B} h_{\nu}^{C},
$$

and the covariant differentiation on $D_{\mu} \Phi_{A}$ has been so determined as the resultant 
expression $D_{\nu}\left(D_{\mu} \Phi_{A}\right)$ transforms like a coadjoint field, that is,

$$
D_{\mu} D_{\nu} \Phi_{A}=\partial_{\mu} D_{\nu} \Phi_{A}+\frac{\partial W_{A B}}{\partial \Phi_{C}} h_{\mu}^{B} D_{\mu} \Phi_{C}
$$

Unfortunately, the curvature does not transform homogeneously because the gauge algebra (2.17) is open:

$$
\delta(c) R_{\mu \nu}^{A}=\frac{\partial W_{B C}}{\partial \Phi_{A}} R_{\mu \nu}^{B} c^{C}+\left\{\left(D_{\mu} \Phi_{D}\right) \frac{\partial^{2} W_{B C}}{\partial \Phi_{D} \partial \Phi_{A}} h_{\nu}^{B} c^{C}-(\mu \leftrightarrow \nu)\right\}
$$

which seems troublesome for the construction of invariant actions.

\subsection{Jacobi Identity for Nonlinear Lie Algebra}

This section is devoted to a brief explanation of an algebraic background for the 'nonlinear' gauge transformation defined in the previous section. The relevant object is the Jacobi identity for nonlinear Lie algebras. ${ }^{[6,11,12]}$

We consider a vector space with a basis $\left\{T_{A}\right\}$ which is the polynomial algebra with a commutative product. On this algebra, we define a bracket product

$$
\left[T_{A}, T_{B}\right]=W_{A B}(T)
$$

as the second product structure, where $W_{A B}(T)$ denotes a polynomial in $T_{C}$ which satisfies the antisymmetry property $W_{A B}=-W_{B A}$ :

$$
W_{A B}(T)=W_{A B}^{(0)}+W_{A B}^{(1) C} T_{C}+W_{A B}^{(2) C D} T_{C} T_{D}+\cdots,
$$

where $W_{A B}^{(0)}, W_{A B}^{(1) C}$, etc. stand for structure constants in this algebra. We assume the bracket product is bilinear and antisymmetric with respect to exchange $A$ with $B$, satisfies the Jacobi identity and has the derivation nature

$$
\begin{aligned}
& {\left[T_{A}, T_{B} T_{C}\right]=\left[T_{A}, T_{B}\right] T_{C}+T_{B}\left[T_{A}, T_{C}\right]} \\
& {\left[T_{A} T_{B}, T_{C}\right]=T_{A}\left[T_{B}, T_{C}\right]+\left[T_{A}, T_{C}\right] T_{B} .}
\end{aligned}
$$

We emphasize that the multiplication of $T_{C}$ and $T_{D}$ is defined in the sense of the polynomial algebra (i.e. not as the matrix, for instance), thus $T_{C} T_{D}=T_{D} T_{C}$. 
Generally speaking, it is not necessary to restrict consideration to the case of polynomial algebra, which is commutative by definition. However, it is enough to treat the commutative case for the present purposes because we make use of the structure functions $W_{A B}$ only in the form $W_{A B}(\Phi)$ where $\Phi$ represents a set of scalar fields $\Phi_{C}$ which do commute: $\Phi_{C} \Phi_{D}=\Phi_{D} \Phi_{C}$.

We note that the zeroth-order term $W_{A B}^{(0)}$ may be recognized as a central element in the algebra (2.23). This definition includes the ordinary Lie algebras as a special case when $W_{A B}(T)$ is linear in $T_{A}$, which makes the algebra (2.23) deserve the name of nonlinear Lie algebra.

The typical examples of nonlinear algebra are $\mathrm{W}$ algebra ${ }^{[14]}$ and quantum group. $^{[15] \star}$ The representation theories of quadratic extension of nonlinear $S U(2)$ algebra and some interesting nonlinear algebras are investigated by several authors. ${ }^{[16]}$

The Jacobi identity with respect to the bracket product (2.23) implies

$$
\frac{\partial W_{[A B}}{\partial T_{D}} W_{C] D}=0
$$

which is a generalization of the Jacobi identity for Lie algebras.

The expression (2.17) can be regarded as a gauge algebra based on the nonlinear Lie algebra (2.23) in the sense that the functions $W_{A B}(\Phi)$ satisfy the same constraint identity (2.6) as the Jacobi identity $(2.25)$ for $W_{A B}(T)$ in the algebra (2.23). However, generally the field $\Phi_{A}$ is not a representation of its algebra different from the coadjoint field of Lie algebra. The relation of $\Phi_{A}$ with the algebra should be more investigated.

\footnotetext{
$\star$ Strictly speaking, it is needed to extend $W_{A B}(T)$ to analytic functions of $T_{A}$ in the definition of nonlinear Lie algebra.
} 


\subsection{An InVARIANt ACtion}

We do not have a straightforward way of constructing invariant actions under the gauge transformation (2.16) owing to the inhomogeneous form of (2.22) as noticed in section 2.1. Hence we will instead seek for an action which provides covariant equations of motion under those transformations, and examine whether it is invariant under them.

We determine of equations of motion as follows: The gauge algebra (2.17) should be closed, at least, on shell so as to originate from some Lagrangian symmetry. Hence the choice

$$
D_{\mu} \Phi_{A}=0
$$

seems an immediate guess for appropriate equations of motion, which are indeed covariant (2.19) under the transformation (2.16).

Since we have the vector, $h_{\mu}^{A}$, and the scalar, $\Phi_{A}$, fields as our basic ones, as a first trial for Lagrangian we consider

$$
\mathcal{L}_{0}=\chi^{\mu \nu} h_{\mu}^{A} D_{\nu} \Phi_{A}
$$

where $\chi^{\mu \nu}$ denotes an invertible constant tensor to be determined later. However, $D_{\nu} \Phi_{A}$ itself contains the fields $h_{\mu}^{A}$, and hence the variation of $\mathcal{L}_{0}$ with respect to $h_{\mu}^{A}$ does not lead to the covariant equations of motion (2.26).

In order to overcome this issue, we modify the Lagrangian into

$$
\mathcal{L}=\mathcal{L}_{0}-\frac{1}{2} \chi^{\mu \nu} W_{A B} h_{\mu}^{A} h_{\nu}^{B}
$$

which yields the desired equations of motion (2.26) provided $\chi^{\mu \nu}=-\chi^{\nu \mu}$. Note that this Lagrangian can be rewritten as

$$
\mathcal{L}=-\frac{1}{2} \chi^{\mu \nu}\left[\Phi_{A} R_{\mu \nu}^{A}+\left(W_{B C}-\Phi_{A} \frac{\partial W_{B C}}{\partial \Phi_{A}}\right) h_{\mu}^{B} h_{\nu}^{C}\right]
$$

up to a total derivative. 
If we require Poincare invariance of the Lagrangian, the antisymmetric tensor $\chi^{\mu \nu}$ should be an invariant one

$$
\chi^{\mu \nu}=\epsilon^{\mu \nu},
$$

where $\epsilon^{\mu \nu}$ denotes the Levi-Civita tensor in two dimensions. At this point, we are inevitably led to considering the two-dimensional situation.

In summary, we have obtained a candidate action

$$
S=\int d^{2} x \mathcal{L} ; \quad \mathcal{L}=-\frac{1}{2} \epsilon^{\mu \nu}\left[\Phi_{A} R_{\mu \nu}^{A}+\left(W_{B C}-\Phi_{A} \frac{\partial W_{B C}}{\partial \Phi_{A}}\right) h_{\mu}^{B} h_{\nu}^{C}\right]
$$

which comes out to be diffeomorphism invariant. In fact, the gauge transformation of $\mathcal{L}$ is

$$
\begin{aligned}
\delta(c) \mathcal{L}= & \partial_{\mu}\left[\epsilon^{\mu \nu}\left(W_{B C}-\Phi_{A} \frac{\partial W_{B C}}{\partial \Phi_{A}}\right) h_{\nu}^{B} c^{C}\right]+\epsilon^{\mu \nu}\left[-\partial_{\mu} W_{B C} h_{\nu}{ }^{B} c^{C}\right. \\
& \left.+\partial_{\mu} \Phi_{A} \frac{\partial W_{B C}}{\partial \Phi_{A}} h_{\nu}{ }^{B} c^{C}+\left(W_{B D} \frac{\partial W_{C E}}{\partial \Phi_{B}}+\frac{1}{2} \frac{\partial W_{C D}}{\partial \Phi_{B}} W_{E B}\right) h_{\mu}{ }^{C} h_{\nu}{ }^{D} c^{E}\right],
\end{aligned}
$$

The relation (2.6) enables us to show

$$
\delta(c) \mathcal{L}=\partial_{\mu}\left[\epsilon^{\mu \nu}\left(W_{B C}-\Phi_{A} \frac{\partial W_{B C}}{\partial \Phi_{A}}\right) h_{\nu}^{B} c^{C}\right]
$$

which confirms the invariance of our action (2.31) under the 'nonlinear' gauge transformation (2.16). The equations of motion which follow from (2.31) are given by the following:

$$
\epsilon^{\mu \nu} D_{\nu} \Phi_{A}=0, \quad \epsilon^{\mu \nu} R_{\mu \nu}^{A}=0,
$$

which are indeed covariant due to the transformation law (2.19), (2.22). 


\subsection{BRS FORMALISM}

Since the action (2.31) has the local gauge symmetry by the gauge transformation (2.16), there are some constraints. In order to quantize the theory in such system, it is necessary to fix gauge. When we make gauge-fixing and quantize gauge theory, use of BRS formalism is standard.

In this section, we formulate BRS formalism of the 'nonlinear' gauge theory.

We discuss how to construct the BRS transformation $\boldsymbol{\delta}_{\mathrm{B}}$ corresponding to the classical symmetry (2.16). Notice that the generator algebra for the gauge transformation (2.16) is open, ${ }^{[17]}$ as can be seen from the gauge algebra (2.17).

We first try to regard the gauge-transformation parameter $c^{A}$ as fermionic FP ghost,

$$
\begin{aligned}
\delta h_{\mu}^{A} & =\partial_{\mu} c^{A}+U_{B C}^{A}(\Phi) h_{\mu}^{B} c^{C}, \\
\delta \Phi_{A} & =W_{B A}(\Phi) c^{B},
\end{aligned}
$$

where $\delta$ is a candidate for the BRS transformation. In this level, $c^{A}$ is not a gauge parameter like $c^{A}$ in the section 2.2, but a fundamental field. With the aid of the BRS nilpotency condition $\delta^{2} \Phi_{A}=0$, we can uniquely determine the transformation law for the ghost whether $c^{A}$ depends on $\Phi_{A}$ or not, which is different from the classical gauge transformation in section 2.1;

$$
\delta c^{A}=-\frac{1}{2} \frac{\partial W_{B C}}{\partial \Phi_{A}} c^{B} c^{C},
$$

which satisfies $\delta^{2} c^{A}=0$. However, the naive definition (2.16) does not satisfy the nilpotency $\delta^{2}=0$ on the field $h_{\mu}^{A}$ owing to the algebra non-closure:

$$
\begin{aligned}
\delta^{2} h_{\mu}^{A} & =-c^{B} c^{C} \frac{\partial^{2} W_{B C}}{\partial \Phi_{A} \partial \Phi_{D}} D_{\mu} \Phi_{D} \\
& =c^{B} c^{C} \frac{\partial^{2} W_{B C}}{\partial \Phi_{A} \partial \Phi_{D}} \epsilon_{\mu \nu} \frac{\delta \mathcal{L}}{\delta h_{\nu}^{D}} .
\end{aligned}
$$

This prevents us from performing simple-minded BRS gauge-fixing of the Lagrangian (2.31). From the general discussion ${ }^{[17]}$, it is sufficient that the BRS trans- 
formation is off-shell symmetry on the quantum action $S_{T}$ :

$$
\delta_{\mathrm{B}} S_{T}=0,
$$

and on-shell nilpotent on all fields under quantum equations of motion because Heisenberg operators always satisfy quantum equations of motion.

The desired BRS transformation $\boldsymbol{\delta}_{\mathrm{B}}$ and gauge-fixed Lagrangian $\mathcal{L}_{T}$ can be obtained as follows: In view of the equation (2.36), we introduce a gauge-fixing fermionic function $\Omega$ to provide

$$
\boldsymbol{\delta}_{\mathrm{B}}=\boldsymbol{\delta}+\boldsymbol{\delta}_{\mathrm{b}},
$$

where we define

$$
\boldsymbol{\delta}_{\mathrm{b}} h_{\mu}^{A}=-i c^{B} c^{C} \frac{\partial^{2} W_{B C}}{\partial \Phi_{A} \partial \Phi_{D}} \epsilon_{\mu \nu} \frac{\delta \Omega}{\delta h_{\nu}^{D}},
$$

and $\boldsymbol{\delta}_{\mathrm{b}}=0$ on the other fields. The gauge-fixed Lagrangian is given by

$$
\mathcal{L}_{T}=\mathcal{L}-i\left(\boldsymbol{\delta}_{\mathrm{B}}-\frac{1}{2} \boldsymbol{\delta}_{\mathrm{b}}\right) \Omega .
$$

Then $\delta_{\mathrm{B}}{ }^{2} h_{\mu}{ }^{A}$ is calculated to be

$$
\begin{aligned}
\boldsymbol{\delta}_{\mathrm{B}}{ }^{2} h_{\mu}{ }^{A}= & c^{B} c^{C} \frac{\partial W_{B C}}{\partial \Phi_{A} \partial \Phi_{D}} \epsilon_{\mu \nu} \frac{\delta}{\delta h_{\nu} D}\left[\mathcal{L}-i\left(\boldsymbol{\delta}_{\mathrm{B}}-\frac{1}{2} \boldsymbol{\delta}_{\mathrm{b}}\right) \Omega\right] \\
& +\frac{\partial^{2}}{\partial \Phi_{A} \partial \Phi_{D}}\left(i c^{B} c^{C} c^{F} \frac{\partial W_{B C}}{\partial \Phi_{E}} W_{E F}\right) \frac{\delta \Omega}{\delta h_{\mu}{ }^{D}}
\end{aligned}
$$

by using

$$
\frac{\delta}{\delta h_{\mu}^{D}}\left[\left(\boldsymbol{\delta}+\frac{1}{2} \boldsymbol{\delta}_{\mathrm{b}}\right) \Omega\right]=\left(\boldsymbol{\delta}+\boldsymbol{\delta}_{\mathrm{b}}\right) \Omega+\frac{\delta \Omega}{\delta h_{\mu} G} \frac{\partial W_{D F}}{\partial \Phi_{G}} c^{F} .
$$

Since the Jacobi identity (2.6) provides the following identity

$$
c^{B} c^{C} c^{F} \frac{\partial W_{B C}}{\partial \Phi_{E}} W_{E F}=0,
$$

it turns out that $\boldsymbol{\delta}_{\mathrm{B}}{ }^{2} h_{\mu}{ }^{A}$ vanishes under quantum equations of motion, that is, $\boldsymbol{\delta}_{\mathrm{B}}$ is on-shell nilpotent, and (2.37) yields a symmetry of the Lagrangian (2.39), as it 
should be:

$$
\begin{aligned}
\boldsymbol{\delta}_{\mathrm{B}} \mathcal{L}_{T}= & \partial_{\mu}\left[\epsilon^{\mu \nu}\left(W_{B C}-\Phi_{A} \frac{\partial W_{B C}}{\partial \Phi_{A}}\right) h_{\nu}^{B} c^{C}\right] \\
& +\partial_{\mu}\left(i \Phi_{A} c^{B} c^{C} \frac{\partial^{2} W_{B C}}{\partial \Phi_{A} \partial \Phi_{D}} \frac{\delta \Omega}{\delta h_{\mu}^{D}}\right) .
\end{aligned}
$$

\subsection{Quantization ON The Cylinder}

By means of the BRS formalism given in the previous section, we quantize the system on the cylindrical spacetime $S^{1} \times \boldsymbol{R}^{1}$ in the temporal gauge.

The gauge-fixing function for the temporal gauge is

$$
\Omega=\bar{c}_{A} h_{0}{ }^{A},
$$

where we introduce FP antighosts $\bar{c}_{A}$ and NL fields $b_{A}$ which satisfy the BRS transformation law

$$
\boldsymbol{\delta}_{\mathrm{B}} \bar{c}_{A}=i b_{A} .
$$

The gauge-fixed Lagrangian (2.39) now reads

$$
\mathcal{L}_{T}=\mathcal{L}+b_{A} h_{0}{ }^{A}+i \bar{c}_{A}\left(\dot{c}^{A}+\frac{\partial W_{B C}}{\partial \Phi_{A}} h_{0}{ }^{B} c^{C}\right)
$$

where the dot denotes a derivative with respect to the time $x^{0}$. This Lagrangian reduces to the form of a free theory

$$
\mathcal{L}_{T}^{\prime}=\Phi_{A} \dot{h}_{1}^{A}+b_{A}^{\prime} h_{0}^{A}+i \bar{c}_{A} \dot{c}^{A}
$$

through field redefinition

$$
b_{A}^{\prime} \equiv b_{A}+\partial_{1} \Phi_{A}-W_{A B} h_{1}^{B}+i \bar{c}_{B} \frac{\partial W_{A C}}{\partial \Phi_{B}} c^{C}
$$

where we have discarded total derivatives w.r.t. $x^{1}$ in the Lagrangian (2.44). 
We can quantize explicitly (2.44) on $S^{1} \times \boldsymbol{R}^{1}$ and derive mode expansion as follows:

$$
\begin{aligned}
h_{1}{ }^{A}(x) & =\frac{1}{\sqrt{2 \pi}} \sum_{n=-\infty}^{\infty} h_{n}{ }^{A} e^{-i n x^{1}}, \\
\Phi_{A}(x) & =\frac{1}{\sqrt{2 \pi}} \sum_{n=-\infty}^{\infty} \Phi_{A n} e^{-i n x^{1}}, \\
c^{A}(x) & =\frac{1}{\sqrt{2 \pi}} \sum_{n=-\infty}^{\infty} c_{n}^{A} e^{-i n x^{1}}, \\
\bar{c}_{A}(x) & =\frac{1}{\sqrt{2 \pi}} \sum_{n=-\infty}^{\infty} \bar{c}_{A n} e^{-i n x^{1}} .
\end{aligned}
$$

From the canonical commutation relations of the fundamental fields, we get the commutation relations of mode variables:

$$
\begin{aligned}
& {\left[\Phi_{A n}, h_{m}^{B}\right]=-i \delta_{A}^{B} \delta_{n+m, 0},} \\
& \left\{\bar{c}_{A n}, c_{m}^{B}\right\}=\delta_{A}^{B} \delta_{n+m, 0} .
\end{aligned}
$$

As usual, it is assumed that $\Phi_{A n}, h_{n}^{A}, \bar{c}_{A n}$ and $c_{n}^{A}$ for $n \geq 1$ annihilate the Fock vacuum. Then nilpotency of the BRS charge in quantum level is necessary for the theory to be unitary and to be well-defined.

The symmetry (2.37) yields a conserved charge

$$
Q=\int_{S^{1}} d x^{1} J_{\mathrm{B}}^{0},
$$

where its Noether current $J_{\mathrm{B}}^{\mu}$ is defined by

$$
\begin{aligned}
J_{\mathrm{B}}^{\mu}= & \boldsymbol{\delta}_{\mathrm{B}} h_{\nu}{ }^{A} \frac{\partial \mathcal{L}_{T}}{\partial\left(\partial_{\mu} h_{\nu}{ }^{A}\right)}+\boldsymbol{\delta}_{\mathrm{B}} \Phi_{A} \frac{\partial \mathcal{L}_{T}}{\partial\left(\partial_{\mu} \Phi_{A}\right)}+\boldsymbol{\delta}_{\mathrm{B}} c^{A} \frac{\partial \mathcal{L}_{T}}{\partial\left(\partial_{\mu} c^{A}\right)}+\boldsymbol{\delta}_{\mathrm{B}} \bar{c}_{A} \frac{\partial \mathcal{L}_{T}}{\partial\left(\partial_{\mu} \bar{c}_{A}\right)}+\boldsymbol{\delta}_{\mathrm{B}} b_{A} \frac{\partial \mathcal{L}_{T}}{\partial\left(\partial_{\mu} b_{A}\right)} \\
& -\epsilon^{\mu \nu}\left(W_{B C}-\Phi_{A} \frac{\partial W_{B C}}{\partial \Phi_{A}}\right) h_{\nu}^{B} c^{C}-i \Phi_{A} c^{B} c^{C} \frac{\partial^{2} W_{B C}}{\partial \Phi_{A} \partial \Phi_{D}} \frac{\delta \Omega}{\delta h_{\mu}^{D}}
\end{aligned}
$$


The zeroth component of its Noether current $J_{\mathrm{B}}$ is given by

$$
\begin{aligned}
J_{\mathrm{B}}^{0} & =\Phi_{A} \partial_{1} c^{A}+J \\
J & =\frac{1}{2}\left\{W_{B C}, h_{1}{ }^{B}\right\} c^{C}-\frac{i}{2} \frac{\partial W_{B C}}{\partial \Phi_{A}} c^{B} \bar{c}_{A} c^{C} .
\end{aligned}
$$

by using (2.42). Note that the operator ordering offers a matter for consideration in the definition of $J$ written by mode variables. The ordering should have been so chosen as the BRS charge (2.46) to be hermitian like (2.47).

This naive definition of the charge suffers from divergence difficulty, which can be removed by normal-ordering prescription of mode variables $Q_{\mathrm{B}}=: Q$ : .

$$
\begin{aligned}
Q_{\mathrm{B}}= & i \sum_{n=-\infty}^{\infty} n: \Phi_{A n} c_{-n}^{A}: \\
& +\frac{1}{2} \sum_{k} \sum_{n_{1}=-\infty}^{\infty} \cdots \sum_{n_{k}=-\infty}^{\infty} \sum_{m=-\infty}^{\infty} W_{B C}^{(k) A_{1} \cdots A_{k}}:\left\{\Phi_{A_{1} n_{1}} \cdots \Phi_{A_{k} n_{k}} h_{1 m}^{B}\right\} c_{-\sum_{k} n_{i}-m}^{C}: \\
& -\frac{i}{2} \sum_{k} \sum_{n_{1}=-\infty}^{\infty} \cdots \sum_{n_{k}=-\infty}^{\infty} \sum_{\substack{m=-\infty \\
l=-\infty}}^{\infty} W_{B C}^{(k) A_{1} \cdots A_{k}}: \Phi_{A_{1} n_{1}} \cdots \Phi_{A_{k-1} n_{k-1}} c_{m}^{B} \bar{c}_{A l} c_{-\sum_{k-1} n_{i}-m-l}^{C}:,
\end{aligned}
$$

The canonical commutation relation (2.45) establishes the nilpotency $Q_{\mathrm{B}}^{2}=0$ of the BRS operator straightforwardly. This enables us to define the quantum theory in terms of the BRS cohomology. ${ }^{[18]}$

\section{3. $R^{2}$ gravity with dynamical torsion}

In this chapter, we consider $R^{2}$ gravity with dynamical torsion in two spacetime dimensions ${ }^{[19]}$ as the typical example of the nonlinear gauge theory. ${ }^{[11]}$ 


\subsection{Pure $R^{2}$ Gravity}

We first make a brief exposition of $R^{2}$ gravity with dynamical torsion in two spacetime dimensions. ${ }^{[19]}$

When the spin connection $\omega_{\mu}^{a b}$ and the zweibein $e_{\mu}^{a}$ are independent variables, the action, invariant under local Lorentz and general coordinate transformations

$$
\begin{aligned}
\delta_{\mathrm{G}} \omega_{\mu} & =\partial_{\mu} \tau-v^{\lambda} \partial_{\lambda} \omega_{\mu}-\left(\partial_{\mu} v^{\lambda}\right) \omega_{\lambda}, \\
\delta_{\mathrm{G}} e_{\mu}{ }^{a} & =-\tau \epsilon^{a b} e_{\mu b}-v^{\lambda} \partial_{\lambda} e_{\mu}{ }^{a}-\left(\partial_{\mu} v^{\lambda}\right) e_{\lambda}{ }^{a},
\end{aligned}
$$

generically ${ }^{\star}$ takes the following form:

$$
S=\int d^{2} x e\left(\frac{1}{16 \alpha} R_{\mu \nu}^{a b} R^{\mu \nu}{ }_{a b}-\frac{1}{8 \beta} T_{\mu \nu}{ }^{a} T_{a}^{\mu \nu}-\gamma\right)
$$

where $\tau$ and $v^{\lambda}$ are local-Lorentz and general coordinate transformation parameters ,and $\alpha, \beta, \gamma$ are constant parameters, and

$$
\begin{aligned}
\omega_{\mu} \epsilon^{a b} & \equiv \omega_{\mu}^{a b}, \quad e \equiv \operatorname{det}\left(e_{\mu}^{a}\right), \\
R_{\mu \nu}^{a b} & \equiv \partial_{\mu} \omega_{\nu}^{a b}-\partial_{\nu} \omega_{\mu}^{a b}, \\
T_{\mu \nu}^{a} & \equiv \partial_{\mu} e_{\nu}^{a}+\omega_{\mu}^{a b} e_{\nu b}-(\mu \leftrightarrow \nu) .
\end{aligned}
$$

Our convention is that the Levi-Civita antisymmetric tensor $\epsilon$ satisfies $\epsilon_{01}=\epsilon^{10}=$ 1 , and the flat metric is given by $\eta=\operatorname{diag}(+1,-1)$.

The Lagrangian in the action (3.2) can be rewritten as

$$
\mathcal{L}_{G}=\frac{1}{4 e \alpha}\left(F_{01}\right)^{2}+\frac{1}{4 e \beta} T_{01}^{a} T_{01 a}-e \gamma
$$

where we have defined

$$
F_{\mu \nu} \equiv \partial_{\mu} \omega_{\nu}-\partial_{\nu} \omega_{\mu}
$$

Various aspects of this theory were extensively studied in Ref.[19].

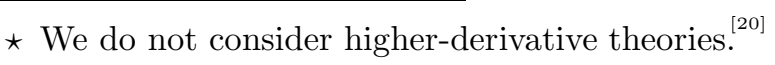




\subsection{YANG-Mills-Like Formulation}

In order to interpret the theory defined by (3.2) as a nonlinear gauge theory, we consider a first-order Lagrangian

$$
\mathcal{L}_{C}=\varphi F_{01}-e \alpha \varphi^{2}+\phi_{a} T_{01}^{a}-e \beta \phi_{a} \phi^{a}-e \gamma
$$

by introducing auxiliary fields $\varphi$ and $\phi_{a}$. This is equivalent to (3.3) in the region $e \neq 0$. Namely, it coincides with the Lagrangian (3.3) when the auxiliary fields $\varphi$ and $\phi_{a}$ are integrated out. The Lagrangian (3.4) also has symmetry under local Lorentz and general coordinate transformations (3.1) with

$$
\begin{aligned}
\delta_{\mathrm{G}} \varphi & =-v^{\lambda} \partial_{\lambda} \varphi \\
\delta_{\mathrm{G}} \phi_{a} & =-\tau \epsilon_{a b} \phi^{b}-v^{\lambda} \partial_{\lambda} \phi_{a} .
\end{aligned}
$$

Its equations of motion are as follows:

$$
\begin{aligned}
& \partial_{\mu} \varphi+\phi_{a} \epsilon^{a b} e_{\mu b}=0, \\
& \partial_{\mu} \phi_{a}+\omega_{\mu} \epsilon_{a b} \phi^{b}-\epsilon_{a b} e_{\mu}^{b}\left(\alpha \varphi^{2}+\beta \phi_{c} \phi^{c}+\gamma\right)=0, \\
& 2 e \alpha \varphi-F_{01}=0, \quad 2 e \beta \phi^{a}-T_{01}^{a}=0 .
\end{aligned}
$$

We note that the Lagrangian (3.4) in a weak-coupling limit $\alpha=\beta=\gamma=0$ is none other than that of topological $\operatorname{ISO}(1,1)$ gauge theory, ${ }^{[9]}$ which is known to be equivalent to the gravity theory ${ }^{[8]}$

$$
I=\int d^{2} x \sqrt{-g} \sigma R
$$

This observation suggests that the full Lagrangian (3.4) itself might have some symmetry of the Yang-Mills type which is related to the $\operatorname{ISO}(1,1)$ gauge transformation. 
In order to find such symmetry, we use a trivial gauge symmetry proportional to the equations of motion (3.6), which Lagrangian system always has. We modify the local-Lorentz and general coordinate transformation (3.1) and (3.5) by the trivial gauge symmetry

$$
\begin{aligned}
\delta \omega_{\mu} & =\delta_{\mathrm{G}} \omega_{\mu}+\epsilon_{\mu \lambda} v^{\lambda}\left[2 \alpha e \varphi-F_{01}\right], \\
\delta e_{\mu}{ }^{a} & =\delta_{\mathrm{G}} e_{\mu}{ }^{a}+\epsilon_{\mu \lambda} v^{\lambda}\left[2 \beta e \phi^{a}-T_{01}{ }^{a}\right], \\
\delta \varphi & =\delta_{\mathrm{G}} \varphi+v^{\lambda}\left[\partial_{\lambda} \varphi+\phi_{a} \epsilon^{a b} e_{\lambda b}\right], \\
\delta \phi_{a} & =\delta_{\mathrm{G}} \phi_{a}+v^{\lambda}\left[\partial_{\lambda} \phi_{a}+\omega_{\lambda} \epsilon_{a b} \phi^{b}-\epsilon_{a b} e_{\lambda}^{b}\left(\alpha \varphi^{2}+\beta \phi_{c} \phi^{c}+\gamma\right)\right] .
\end{aligned}
$$

If we set

$$
t=\tau-v^{\lambda} \omega_{\lambda}, \quad c^{a}=-v^{\lambda} e_{\lambda}^{a}
$$

we can explicitly write down ${ }^{\star}$ the symmetry $\delta$ :

$$
\begin{aligned}
\delta \omega_{\mu} & =\partial_{\mu} t+2 \alpha \epsilon_{b c} c^{b} e_{\mu}^{c} \varphi \\
\delta e_{\mu}{ }^{a} & =-t \epsilon^{a b} e_{\mu b}+\partial_{\mu} c^{a}+\omega_{\mu} \epsilon^{a b} c_{b}+2 \beta \epsilon_{b c} c^{b} e_{\mu}{ }^{c} \phi^{a}, \\
\delta \varphi & =\epsilon^{a b} c_{a} \phi_{b}, \\
\delta \phi_{a} & =-t \epsilon_{a b} \phi^{b}+\epsilon_{a b} c^{b}\left(\alpha \varphi^{2}+\beta \phi_{c} \phi^{c}+\gamma\right),
\end{aligned}
$$

which reduces to the $\operatorname{ISO}(1,1)$ gauge transformation ${ }^{\dagger}$ when $\alpha=\beta=\gamma=0$. It is straightforward to see

$$
\delta \mathcal{L}_{C}=\partial_{\mu}\left[\epsilon^{\mu \nu} e_{\nu}^{a} \epsilon_{a b} c^{b}\left(\alpha \varphi^{2}+\beta \phi_{c} \phi^{c}-\gamma\right)\right]
$$

The gauge transformations (3.1), (3.5), and (3.9) are reducible.

In the following sections, we confirm (3.9) is a special case of our nonlinear gauge transformation introduced in the previous chapter.

* Note added: Quite recently, this symmetry has been also obtained in Ref.[21] by means of Hamiltonian analysis of the action (3.2).

$\dagger$ Instead of the Poincaré group $\operatorname{ISO}(1,1)$, we can deal with the de Sitter group $\mathrm{SO}(2,1)$ by means of a constant shift in the field $\varphi$ and an appropriate redefinition of the parameter $\gamma$. 


\subsection{Quadratically Nonlinear Poincaré Algebra}

The general-covariance property of our action (2.31) suggests that we might utilize it to construct two-dimensional gravitation theory. It seems a natural choice to adopt the Poincaré algebra in two dimensions as a base algebra on which the construction will be performed. Since the diffeomorphism invariance is guaranteed by the definition of (2.31), our requirement is that the theory should be localLorentz covariant. Hence we consider quadratically nonlinear extension of the Poincaré algebra which preserves the Lorentz structure of the genuine Poincaré algebra:

$$
\begin{aligned}
& {[J, J]=0, \quad\left[J, P_{a}\right]=\epsilon_{a}{ }^{b} P_{b},} \\
& {\left[P_{a}, P_{b}\right]=-\epsilon_{a b}\left(\alpha J J+\beta \eta^{c d} P_{c} P_{d}+\gamma I\right),}
\end{aligned}
$$

where $\alpha, \beta, \gamma$ are constants, $\eta^{c d}$ is the two-dimensional flat metric, and $I$ is a central element added to the Poincaré algebra.

We set $\left\{T_{A}\right\}=\left\{P_{a}, J\right\}$, that is, $T_{0}=P_{0}, T_{1}=P_{1}$, and $T_{2}=J$. Then the structure constants defined for the above algebra are given by

$$
\begin{aligned}
W_{C D}^{(1) a} & =\epsilon^{a}{ }_{b}\left(\delta_{C}{ }^{b} \delta_{D}{ }^{2}-\delta_{C}{ }^{2} \delta_{D}^{b}\right), \quad W_{C D}^{(1) 2}=0, \\
W_{C D}^{(2) 22} & =-\alpha\left(\delta_{C}{ }^{k} \delta_{D}{ }^{l} \epsilon_{k l}\right), \quad W_{C D}^{(2) a b}=-\beta \eta^{a b}\left(\delta_{C}{ }^{k} \delta_{D}{ }^{l} \epsilon_{k l}\right), \quad W_{C D}^{(2) 2 a}=W_{C D}^{(2) a 2}=0, \\
W_{C D}^{(0)} & =-\gamma\left(\delta_{C}{ }^{k} \delta_{D}{ }^{l} \epsilon_{k l}\right), \\
W^{(i)} & =0 \quad \text { for } i \geq 3,
\end{aligned}
$$

where the notation of $(2.24)$ is used.

We also set gauge fields $h_{\mu}^{A}=\left(e_{\mu}^{a}, \omega_{\mu}\right)$ and scalar fields $\Phi_{A}=\left(\phi_{a}, \varphi\right)$ to obtain $(3.4)$ :

$$
\mathcal{L}_{1}=-\frac{1}{2} \epsilon^{\mu \nu} \varphi F_{\mu \nu}-\frac{1}{2} \epsilon^{\mu \nu} \phi_{a} T_{\mu \nu}^{a}-e \alpha \varphi^{2}-e \beta \phi_{a} \phi^{a}-e \gamma
$$

\footnotetext{
$\ddagger$ The right-hand side of $\left[P_{a}, P_{b}\right]$ can contain a term proportional to $\epsilon_{a b} J$, which results in nonlinear (anti-)de Sitter algebra. We omit that term just for simplicity.
} 
as the Lagrangian (2.31). The gauge transformation law (2.16) now reads (3.9):

$$
\begin{aligned}
\delta \omega_{\mu} & =\partial_{\mu} t+2 \alpha \epsilon_{b c} c^{b} e_{\mu}^{c} \varphi \\
\delta e_{\mu}{ }^{a} & =-t \epsilon^{a b} e_{\mu b}+\partial_{\mu} c^{a}+\omega_{\mu} \epsilon^{a b} c_{b}+2 \beta \epsilon_{b c} c^{b} e_{\mu}{ }^{c} \phi^{a}, \\
\delta \varphi & =\epsilon^{a b} c_{a} \phi_{b}, \\
\delta \phi_{a} & =-t \epsilon_{a b} \phi^{b}+\epsilon_{a b} c^{b}\left(\alpha \varphi^{2}+\beta \phi_{c} \phi^{c}+\gamma\right),
\end{aligned}
$$

where we have put $c^{A}=\left(c^{a}, t\right)$.

This theory completely coincides with the Yang-Mills-like formulation of $R^{2}$ gravity with dynamical torsion we proposed the previous section. Note that the

gravitation theory (3.2) is obtained if one integrates out the scalar fields $\varphi$ and $\phi_{a}$ in the Lagrangian (3.4) under the condition $e \neq 0$. In short, two-dimensional $R^{2}$ gravity with dynamical torsion has been shown to be a gauge theory based on quadratically nonlinear Poincaré algebra.

\section{Generic Form of 'Dilaton' Gravity}

In this chapter, we consider generic form of 'dilaton' gravity as the nonlinear gauge theory. ${ }^{[12]}$

\subsection{The ACtion}

The general expression for the action of two-dimensional metric $\bar{g}_{\mu \nu}$ coupled to a scalar field $\bar{\varphi}$ (without higher-derivative terms) is given by

$$
S=\int d^{2} x \sqrt{-\bar{g}}\left(\frac{1}{2} \bar{g}^{\mu \nu} \partial_{\mu} \bar{\varphi} \partial_{\nu} \bar{\varphi}+\frac{1}{2} \mathcal{U}(\bar{\varphi}) \bar{R}-\mathcal{V}(\bar{\varphi})\right)
$$

We may replace $\mathcal{U}(\bar{\varphi})$ by a linear function ${ }^{[22]}$

$$
S=\int d^{2} x \sqrt{-g}\left(\frac{\kappa}{2} g^{\mu \nu} \partial_{\mu} \varphi \partial_{\nu} \varphi+\frac{1}{2} \varphi R-\mathcal{W}(\varphi)\right)
$$


through a field redefinition ${ }^{\S}$

$$
\begin{aligned}
& \varphi=\mathcal{U}(\bar{\varphi}), \quad g_{\mu \nu}=e^{\rho} \bar{g}_{\mu \nu}, \quad \mathcal{W}(\varphi)=e^{\rho} \mathcal{V}(\bar{\varphi}) \\
& \rho(\bar{\varphi}) \equiv-\kappa \mathcal{U}(\bar{\varphi})+\int d \bar{\varphi}\left(\frac{\partial \mathcal{U}}{\partial \bar{\varphi}}\right)^{-1}
\end{aligned}
$$

where $\kappa$ is an arbitrary constant. In particular, we can set $\kappa=0$ to obtain

$$
S=\int d^{2} x \mathcal{L}_{D} ; \quad \mathcal{L}_{D}=\sqrt{-g}\left(\frac{1}{2} \varphi R-\mathcal{W}(\varphi)\right)
$$

The action (4.4) is of course invariant under the following general coordinate transformation:

$$
\begin{aligned}
\delta_{\mathrm{G}} g_{\mu \nu} & =-v^{\lambda} \partial_{\lambda} g_{\mu \nu}-\left(\partial_{\mu} v^{\lambda}\right) g_{\lambda \nu}-\left(\partial_{\nu} v^{\lambda}\right) g_{\mu \lambda}, \\
\delta_{\mathrm{G}} \varphi & =-v^{\lambda} \partial_{\lambda} \varphi .
\end{aligned}
$$

In the following section, we investigate a gauge-theoretical origin of the 'dilaton' field $\varphi$ in the action (4.4), which might be regarded as a "pure" gravity in two dimensions.

\subsection{General Nonlinear Poincaré Algebra}

It seems a natural choice to adopt the Poincaré algebra in two dimensions as a base algebra on which the construction will be performed. Since the diffeomorphism invariance is guaranteed by the definition of (2.31), our requirement is that the theory should be local-Lorentz covariant. Hence we consider nonlinear extension "of the Poincaré algebra which preserves the Lorentz structure of the genuine

$\S$ This field transformation is well-defined only locally in the field space of $\bar{\varphi}$ where $(\partial \mathcal{U} / \partial \bar{\varphi}) \neq$ 0 . In particular, it is inapplicable to the case of $\mathcal{U}(\bar{\varphi})=$ constant, where the field $\bar{\varphi}$ is regarded as a scalar matter rather than the 'dilaton' field.

- The following form of nonlinear Poincaré algebra is not the most general one that preserves the Lorentz structure. It is chosen so as to realize the torsion-free condition as an equation of motion in the resultant 'nonlinear' gauge theory. 
Poincaré algebra:

$$
\begin{aligned}
& {[J, J]=0, \quad\left[J, P_{a}\right]=\epsilon_{a b} \eta^{b c} P_{c}} \\
& {\left[P_{a}, P_{b}\right]=-\epsilon_{a b} \mathcal{W}(J)}
\end{aligned}
$$

where $\eta^{c d}$ is the two-dimensional Minkowski metric. Note that the choice $\mathcal{W}(J)=0$ corresponds to the original Poincaré algebra.

We set $\left\{T_{A}\right\}=\left\{P_{a}, J\right\}$, that is, $T_{0}=P_{0}, T_{1}=P_{1}$, and $T_{2}=J$. Then the structure functions defined in (2.23) for the above algebra are given by

$$
\begin{aligned}
& W_{2 a}=-W_{a 2}=\epsilon_{a b} \eta^{b c} P_{c}, \quad W_{22}=0 \\
& W_{a b}=-\epsilon_{a b} \mathcal{W}(J) .
\end{aligned}
$$

We also set the vector field $h_{\mu}^{A}=\left(e_{\mu}{ }^{a}, \omega_{\mu}\right)$ and the scalar field $\Phi_{A}=\left(\phi_{a}, \varphi\right)$ to obtain

$$
\mathcal{L}_{S}=-\frac{1}{2} \epsilon^{\mu \nu} \varphi F_{\mu \nu}-\frac{1}{2} \epsilon^{\mu \nu} \phi_{a} T_{\mu \nu}^{a}-e \mathcal{W}(\varphi)
$$

as the Lagrangian (2.31), where we have defined

$$
\begin{aligned}
F_{\mu \nu} & \equiv \partial_{\mu} \omega_{\nu}-\partial_{\nu} \omega_{\mu}, \\
T_{\mu \nu}^{a} & \equiv \partial_{\mu} e_{\nu}^{a}+\omega_{\mu} \epsilon^{a b} e_{\nu b}-(\mu \leftrightarrow \nu), \\
e & \equiv \operatorname{det}\left(e_{\mu}{ }^{a}\right) .
\end{aligned}
$$

The gauge transformation law (2.16) now reads

$$
\begin{aligned}
\delta \omega_{\mu} & =\partial_{\mu} t+\epsilon_{b c} c^{b} e_{\mu}{ }^{c} \frac{\partial \mathcal{W}(\varphi)}{\partial \varphi}, \\
\delta e_{\mu}{ }^{a} & =-t \epsilon^{a b} e_{\mu b}+\partial_{\mu} c^{a}+\omega_{\mu} \epsilon^{a b} c_{b}, \\
\delta \varphi & =\epsilon^{a b} c_{a} \phi_{b} \\
\delta \phi_{a} & =-t \epsilon_{a b} \phi^{b}+\epsilon_{a b} c^{b} \mathcal{W}(\varphi),
\end{aligned}
$$

where we have put $c^{A}=\left(c^{a}, t\right)$. 
We can actually confirm

$$
\delta \mathcal{L}_{S}=-\partial_{\mu}\left[\epsilon^{\mu \nu} e_{\nu}^{a} \epsilon_{a b} c^{b}\left(\mathcal{W}-\varphi \frac{\partial \mathcal{W}}{\partial \varphi}\right)\right]
$$

which corresponds to (2.32) in the previous section. The equations of motion which follow from (4.8) are given by

$$
\begin{aligned}
& \partial_{\mu} \varphi+\phi_{a} \epsilon^{a b} e_{\mu b}=0, \\
& \partial_{\mu} \phi_{a}+\omega_{\mu} \epsilon_{a b} \phi^{b}-\epsilon_{a b} e_{\mu}^{b} \mathcal{W}=0, \\
& \frac{1}{2} \epsilon^{\mu \nu} F_{\mu \nu}+e \frac{\partial \mathcal{W}}{\partial \varphi}=0, \quad \frac{1}{2} \epsilon^{\mu \nu} T_{\mu \nu}^{a}=0,
\end{aligned}
$$

which of course corresponds to (2.33).

Note that the gravitation theory (4.4) is obtained if one puts $e_{\mu}{ }^{a} e_{\nu}{ }^{b} \eta_{a b}=g_{\mu \nu}$ and integrates out the fields $\phi_{a}$ and $\omega_{\mu}$ in the Lagrangian (4.8) under the condition $e \neq 0$. The reformulation (4.8) reveals that the theory (4.4) possesses hidden gauge symmetry (4.10) of the Yang-Mills type. This gauge symmetry includes diffeomorphism, as is the case for the Yang-Mills-like formulation of $R^{2}$ gravity with dynamical torsion in the previous chapter.

\section{5. 'Nonlinear' Supergauge Theory}

In this chapter, we extend the previous consideration to the a 'nonlinear' supergauge theory i. e. gauge theory based on nonlinear extension of Lie superalgebras, and apply this gauge theory to two-dimensional dilaton supergravity. 


\section{1. 'Nonlinear' Gauge Transformation}

We introduce a supermultiplet of gauge fields $\left(h_{\mu}^{A}, \xi_{\mu}{ }^{\alpha}\right)$, where $h_{\mu}^{A}$ is a bosonic field and $\xi_{\mu}{ }^{\alpha}$ a fermionic one. $A$ denotes an internal index of the even generators and $\alpha$ the odd generators. As is the case for 'nonlinear' gauge theory exposed in Ref.[6, 11, 12], we need a 'coadjoint' bosonic scalar field $\phi_{A}$ and a fermionic one $\psi_{\alpha}$.

According to the same discussion as in Ref. [12], we determine the gauge transformation of gauge fields $h_{\mu}^{A}$ and $\xi_{\mu}^{\alpha}$, auxiliary fields $\phi_{A}$ and $\psi_{\alpha}$.

By the same discussion as the bosonic case in the chapter 2, the gauge transformation of $h_{\mu}{ }^{A}, \xi_{\mu}{ }^{\alpha}, \phi_{A}$ and $\psi_{\alpha}$ is determined. The resulting form of the gauge transformation $\delta(c, \tau)$ is given by

$$
\begin{aligned}
& \delta(c, \tau) h_{\mu}{ }^{A}=\partial_{\mu} c^{A}-c^{B}\left(\frac{\partial W_{B C}}{\partial \phi_{A}} h_{\mu}{ }^{C}+i \frac{\partial U_{B \gamma}}{\partial \phi_{A}} \xi_{\mu}{ }^{\gamma}\right)-i \tau^{\beta}\left(\frac{\partial U_{C \beta}}{\partial \phi_{A}} h_{\mu}{ }^{C}+\frac{\partial V_{\beta \gamma}}{\partial \phi_{A}} \xi_{\mu}{ }^{\gamma}\right) \\
& \delta(c, \tau) \xi_{\mu}{ }^{\alpha}=\partial_{\mu} \tau^{\alpha}+c^{B}\left(-i \frac{\partial W_{B C}}{\partial \psi_{\alpha}} h_{\mu}{ }^{C}-\frac{\partial U_{B \gamma}}{\partial \psi_{\alpha}} \xi_{\mu}{ }^{\gamma}\right)+\tau^{\beta}\left(\frac{\partial U_{C \beta}}{\partial \psi_{\alpha}} h_{\mu}{ }^{C}+\frac{\partial V_{\beta \gamma}}{\partial \psi_{\alpha}} \xi_{\mu}^{\gamma}\right) \\
& \delta(c, \tau) \phi_{A}=c^{B} W_{B A}+i \tau^{\beta} U_{A \beta}, \\
& \delta(c, \tau) \psi_{\alpha}=c^{B} U_{B \alpha}+\tau^{\beta} V_{\beta \alpha},
\end{aligned}
$$

where $c^{A}, \tau^{\alpha}$ are gauge parameters and $W_{A B}(\phi, \psi), U_{A \beta}(\phi, \psi)$ and $V_{\alpha \beta}(\phi, \psi)$ are smooth functions of the fields $\phi_{A}$ and $\psi_{\alpha}$ to be determined below. Since the transformation includes the one in the case of the usual Lie superalgebra, we assume that $W_{A B}(\phi, \psi)=-W_{B A}(\phi, \psi)$ and $V_{\alpha \beta}(\phi, \psi)=V_{\beta \alpha}(\phi, \psi)$. Similar to $(2.6), W_{A B}$, $U_{A \beta}$ and $V_{\alpha \beta}$ should satisfy the condition 


$$
\begin{aligned}
& \frac{\partial W_{A B}}{\partial \phi_{D}} W_{C D}+U_{A \delta} \frac{\partial W_{B C}}{\partial \psi_{\delta}}+\operatorname{Antisym}(A, B, C)=0, \\
& \frac{\partial W_{A B}}{\partial \phi_{D}} U_{D \gamma}+\frac{\partial U_{B \gamma}}{\partial \phi_{D}} W_{D A}-\frac{\partial U_{A \gamma}}{\partial \phi_{D}} W_{D B}+U_{B \delta} \frac{\partial U_{A \gamma}}{\partial \psi_{\delta}}-U_{A \delta} \frac{\partial U_{B \gamma}}{\partial \psi_{\delta}}-i V_{\gamma \delta} \frac{\partial W_{A B}}{\partial \psi_{\delta}}=0, \\
& \frac{\partial V_{\beta \gamma}}{\partial \phi_{D}} W_{D A}+i U_{D \gamma} \frac{\partial U_{A \beta}}{\partial \phi_{D}}+i U_{D \beta} \frac{\partial U_{A \gamma}}{\partial \phi_{D}}+V_{\beta \delta} \frac{\partial U_{A \gamma}}{\partial \psi_{\delta}}+V_{\gamma \delta} \frac{\partial U_{A \beta}}{\partial \psi_{\delta}}+\frac{\partial V_{\beta \gamma}}{\partial \psi_{\delta}} U_{A \delta}=0, \\
& i \frac{\partial V_{\alpha \beta}}{\partial \phi_{D}} U_{D \gamma}+V_{\gamma \delta} \frac{\partial V_{\alpha \beta}}{\partial \psi_{\delta}}+\operatorname{Sym}(\alpha, \beta, \gamma)=0,
\end{aligned}
$$

where $\operatorname{Antisym}(A, B, C)$ denotes antisymmetrization in the indices $A, B$ and $C$, and

$\operatorname{Sym}(\alpha, \beta, \gamma)$ symmetrization in the indices $\alpha, \beta$ and $\gamma$. The expression (5.2) can be derived from the Jacobi identity for a nonlinear Lie superalgebra with structure functions $W_{A B}, U_{A \beta}$ and $V_{\alpha \beta}$.

The gauge algebra is given by

$$
\begin{aligned}
{\left[\delta\left(c_{1}, \tau_{1}\right), \delta\left(c_{2}, \tau_{2}\right)\right] h_{\mu}{ }^{A}=} & \delta\left(c_{3}, \tau_{3}\right) h_{\mu}{ }^{A}-c_{1}^{B} c_{2}^{C}\left(D_{\mu} \phi_{D} \frac{\partial^{2} W_{B C}}{\partial \phi_{A} \partial \phi_{D}}+D_{\mu} \psi_{\delta} \frac{\partial^{2} W_{B C}}{\partial \phi_{A} \partial \psi_{\delta}}\right) \\
& +i\left(\tau_{2}^{B} c_{1}^{\gamma}-\tau_{1}^{\gamma} c_{2}^{B}\right)\left(D_{\mu} \phi_{D} \frac{\partial^{2} U_{B \gamma}}{\partial \phi_{A} \partial \phi_{D}}+D_{\mu} \psi_{\delta} \frac{\partial^{2} U_{B \gamma}}{\partial \phi_{A} \partial \psi_{\delta}}\right) \\
& -i \tau_{1}^{\beta} \tau_{2}^{\gamma}\left(D_{\mu} \phi_{D} \frac{\partial^{2} V_{\beta \gamma}}{\partial \phi_{A} \partial \phi_{D}}+D_{\mu} \psi_{\delta} \frac{\partial^{2} V_{\beta \gamma}}{\partial \phi_{A} \partial \psi_{\delta}}\right), \\
{\left[\delta\left(c_{1}, \tau_{1}\right), \delta\left(c_{2}, \tau_{2}\right)\right] \xi_{\mu}{ }^{\alpha}=} & \delta\left(c_{3}, \tau_{3}\right) \xi_{\mu}{ }^{\alpha}+i c_{1}^{B} c_{2}^{C}\left(D_{\mu} \phi_{D} \frac{\partial^{2} W_{B C}}{\partial \psi_{\alpha} \partial \phi_{D}}+D_{\mu} \psi_{\delta} \frac{\partial^{2} W_{B C}}{\partial \psi_{\delta} \partial \psi_{\alpha}}\right) \\
& -\left(c_{1}^{B} \tau_{2}^{\gamma}-c_{2}^{B} \tau_{1}^{\gamma}\right)\left(D_{\mu} \phi_{D} \frac{\partial^{2} U_{B \gamma}}{\partial \psi_{\alpha} \partial \phi_{D}}+D_{\mu} \psi_{\delta} \frac{\partial^{2} U_{B \gamma}}{\partial \psi_{\delta} \partial \psi_{\alpha}}\right) \\
& -\tau_{1}^{\beta} \tau_{2}^{\gamma}\left(D_{\mu} \phi_{D} \frac{\partial^{2} V_{\beta \gamma}}{\partial \psi_{\alpha} \partial \phi_{D}}+D_{\mu} \psi_{\delta} \frac{\partial^{2} V_{\beta \gamma}}{\partial \psi_{\delta} \partial \psi_{\alpha}}\right), \\
{\left[\delta\left(c_{1}, \tau_{1}\right), \delta\left(c_{2}, \tau_{2}\right)\right] \phi_{A}=} & \delta\left(c_{3}, \tau_{3}\right) \phi_{A}, \\
{\left[\delta\left(c_{1}, \tau_{1}\right), \delta\left(c_{2}, \tau_{2}\right)\right] \psi_{\alpha}=} & \delta\left(c_{3}, \tau_{3}\right) \psi_{\alpha},
\end{aligned}
$$

where we have defined

$$
\begin{aligned}
& D_{\mu} \phi_{A} \equiv \partial_{\mu} \phi_{A}+W_{A B} h_{\mu}^{B}+i U_{A \beta} \xi_{\mu}{ }^{\beta} \\
& D_{\mu} \psi_{\alpha} \equiv \partial_{\mu} \psi_{\alpha}-U_{B \alpha} h_{\mu}{ }^{B}-V_{\alpha \beta} \xi_{\mu}{ }^{\beta}
\end{aligned}
$$


and the composite parameters $c_{3}$ and $\tau_{3}$ become

$$
\begin{aligned}
c_{3}^{D} & \equiv c_{1}^{B} c_{2}^{C} \frac{\partial W_{B C}}{\partial \phi_{D}}-i\left(c_{1}^{B} \tau_{2}^{\gamma}-c_{2}^{B} \tau_{1}^{\gamma}\right) \frac{\partial U_{B \gamma}}{\partial \phi_{D}}+i \tau_{1}^{\beta} \tau_{2}^{\gamma} \frac{\partial V_{\beta \gamma}}{\partial \phi_{D}} \\
\tau_{3}^{\delta} & \equiv-i c_{1}^{B} c_{2}^{C} \frac{\partial W_{B C}}{\partial \psi_{\delta}}+\left(c_{1}^{B} \tau_{2}^{\gamma}-c_{2}^{B} \tau_{1}^{\gamma}\right) \frac{\partial U_{B \gamma}}{\partial \psi_{\delta}}+\tau_{1}^{\beta} \tau_{2}^{\gamma} \frac{\partial V_{\beta \gamma}}{\partial \psi_{\delta}}
\end{aligned}
$$

$D_{\mu} \phi_{A}$ and $D_{\mu} \psi_{\alpha}$ transform as coadjoint vectors, i. e.

$$
\begin{aligned}
& \delta(c, \tau)\left(D_{\mu} \phi_{A}\right)=c^{B}\left[\frac{\partial W_{B A}}{\partial \phi_{C}} D_{\mu} \phi_{C}-\frac{\partial W_{B A}}{\partial \psi_{\gamma}} D_{\mu} \psi_{\gamma}\right]+i \tau^{\beta}\left[\frac{\partial U_{A \beta}}{\partial \phi_{C}} D_{\mu} \phi_{C}+\frac{\partial U_{A \beta}}{\partial \psi_{\gamma}} D_{\mu} \psi_{\gamma}\right] \\
& \delta(c, \tau)\left(D_{\mu} \psi_{\alpha}\right)=c^{B}\left[\frac{\partial U_{B \alpha}}{\partial \phi_{C}} D_{\mu} \phi_{C}+\frac{\partial U_{B \alpha}}{\partial \psi_{\gamma}} D_{\mu} \psi_{\gamma}\right]+\tau^{\beta}\left[\frac{\partial V_{\beta \alpha}}{\partial \phi_{C}} D_{\mu} \phi_{C}-\frac{\partial V_{\beta \alpha}}{\partial \psi_{\gamma}} D_{\mu} \psi_{\gamma}\right]
\end{aligned}
$$

Thus $D_{\mu}$ is recognized as a covariant differentiation.

The commutators of two covariant differentiations provide

$$
\begin{aligned}
& {\left[D_{\mu}, D_{\nu}\right] \phi_{A}=W_{A B} R_{\mu \nu}^{B}+i U_{A \beta} R_{\mu \nu}^{\beta}} \\
& {\left[D_{\mu}, D_{\nu}\right] \psi_{\alpha}=-U_{B \alpha} R_{\mu \nu}^{B}-V_{\beta \alpha} R_{\mu \nu}^{\beta}}
\end{aligned}
$$

where the curvatures $R_{\mu \nu}^{A}$ and $R_{\mu \nu}^{\alpha}$ are defined by

$$
\begin{aligned}
& R_{\mu \nu}^{A}=\partial_{\mu} h_{\nu}{ }^{A}-\partial_{\nu} h_{\mu}{ }^{A}+\frac{\partial W_{B C}}{\partial \phi_{A}} h_{\mu}{ }^{B} h_{\nu}{ }^{C}+i \frac{\partial U_{B \gamma}}{\partial \phi_{A}}\left(h_{\mu}{ }^{B} \xi_{\nu}{ }^{\gamma}-h_{\nu}{ }^{B} \xi_{\mu}{ }^{\gamma}\right)+i \frac{\partial V_{\beta \gamma}}{\partial \phi_{A}} \xi_{\mu}{ }^{\beta} \xi_{\nu}{ }^{\gamma}, \\
& R_{\mu \nu}^{\alpha}=\partial_{\mu} \xi_{\nu}{ }^{\alpha}-\partial_{\nu} \xi_{\mu}{ }^{\alpha}-i \frac{\partial W_{B C}}{\partial \psi_{\alpha}} h_{\mu}{ }^{B} h_{\nu}{ }^{C}+\frac{\partial U_{B \gamma}}{\partial \psi_{\alpha}}\left(h_{\mu}{ }^{B} \xi_{\nu}{ }^{\gamma}-h_{\nu}{ }^{B} \xi_{\mu}{ }^{\gamma}\right)+\frac{\partial V_{\beta \gamma}}{\partial \psi_{\alpha}} \xi_{\mu}{ }^{\beta} \xi_{\nu}{ }^{\gamma}
\end{aligned}
$$

and the covariant differentiations on $D_{\mu} \phi_{A}$ and $D_{\mu} \psi_{\alpha}$ have been determined in such a way that the resultant expressions $D_{\nu}\left(D_{\mu} \phi_{A}\right)$ and $D_{\nu}\left(D_{\mu} \psi_{\alpha}\right)$ transform like coadjoint fields. The curvatures do not transform homogeneously. 


\subsection{AN INVARIANT ACTION}

We do not have a straightforward way of constructing invariant actions under the gauge transformation (5.1), as noticed from the inhomogeneous form of the transforms of the curvatures. Hence by similar discussion to chapter 2, we will instead seek an action which provides covariant equations of motion under those transformations, and examine whether it is invariant under them.

The gauge algebra (5.3) should be closed, at least, on shell so as to originate from some Lagrangian symmetry. Hence the choice

$$
D_{\mu} \phi_{A}=0, \quad D_{\mu} \psi_{\alpha}=0,
$$

seems an immediate guess for appropriate equations of motion, which are indeed covariant (5.6) under the transformation (5.1). If $W_{A B}, U_{A \beta}$ and $V_{\beta \alpha}$ are nondegenerate, the equations (5.9) imply

$$
R_{\mu \nu}^{A}=0, \quad R_{\mu \nu}^{\alpha}=0
$$

under the formula (5.7).

The Lagrangian is constructed from our basic fields which are the vector ones $h_{\mu}^{A}$ and $\xi_{\mu}{ }^{\alpha}$ the scalar ones $\phi_{A}$ and $\psi_{\alpha}$. Moreover since it is natural that the desired Lagrangian produces (5.9) and (5.10) as the equations of motion, we are led to the Lagrangian

$$
\begin{aligned}
\mathcal{L}= & -\frac{1}{2} \chi^{\mu \nu}\left[\phi_{A} R_{\mu \nu}^{A}+i \psi_{\alpha} R_{\mu \nu}^{\alpha}+\left(W_{B C}-\phi_{A} \frac{\partial W_{B C}}{\partial \phi_{A}}-\psi_{\alpha} \frac{\partial W_{B C}}{\partial \psi_{\alpha}}\right) h_{\mu}{ }^{B} h_{\nu}{ }^{C}\right. \\
& \left.+2 i\left(U_{B \gamma}-\phi_{A} \frac{\partial U_{B \gamma}}{\partial \phi_{A}}-\psi_{\alpha} \frac{\partial U_{B \gamma}}{\partial \psi_{\alpha}}\right) h_{\mu}{ }^{B} \xi_{\nu}{ }^{\gamma}+i\left(V_{\beta \gamma}-\phi_{A} \frac{\partial V_{\beta \gamma}}{\partial \phi_{A}}-\psi_{\alpha} \frac{\partial V_{\beta \gamma}}{\partial \psi_{\alpha}}\right) \xi_{\mu}{ }^{\beta} \xi_{\nu}{ }^{\gamma}\right]
\end{aligned}
$$

which indeed yields the equations of motion (5.9) and (5.10), where $\chi^{\mu \nu}=-\chi^{\nu \mu}$. 
If we require Poincare invariance of the Lagrangian, the antisymmetric tensor $\chi^{\mu \nu}$ should be an invariant one

$$
\chi^{\mu \nu}=\epsilon^{\mu \nu},
$$

where $\epsilon^{\mu \nu}$ denotes the Levi-Civita tensor in two dimensions. At this point, we are led to considering the two-dimensional situation.

In summary, we have obtained a candidate action

$$
\begin{aligned}
S= & \int d^{2} x \mathcal{L}, \\
\mathcal{L}= & -\frac{1}{2} \epsilon^{\mu \nu}\left[\phi_{A} R_{\mu \nu}^{A}+i \psi_{\alpha} R_{\mu \nu}^{\alpha}+\left(W_{B C}-\phi_{A} \frac{\partial W_{B C}}{\partial \phi_{A}}-\psi_{\alpha} \frac{\partial W_{B C}}{\partial \psi_{\alpha}}\right) h_{\mu}{ }^{B} h_{\nu}{ }^{C}\right. \\
& +i\left(U_{B \gamma}-\phi_{A} \frac{\partial U_{B \gamma}}{\partial \phi_{A}}-\psi_{\alpha} \frac{\partial U_{B \gamma}}{\partial \psi_{\alpha}}\right)\left(h_{\mu}{ }^{B} \xi_{\nu}{ }^{\gamma}-h_{\nu}{ }^{B} \xi_{\mu}{ }^{\gamma}\right) \\
& \left.+i\left(V_{\beta \gamma}-\phi_{A} \frac{\partial V_{\beta \gamma}}{\partial \phi_{A}}-\psi_{\alpha} \frac{\partial V_{\beta \gamma}}{\partial \psi_{\alpha}}\right) \xi_{\mu}{ }^{\beta} \xi_{\nu}{ }^{\gamma}\right], \\
= & -\frac{1}{2} \epsilon^{\mu \nu}\left[\phi_{A}\left(\partial_{\mu} h_{\nu}{ }^{A}-\partial_{\nu} h_{\mu}{ }^{A}\right)+W_{B C} h_{\mu}{ }^{B} h_{\nu}{ }^{C}\right] \\
& -\frac{1}{2} \epsilon^{\mu \nu}\left[i \psi_{\alpha}\left(\partial_{\mu} \xi_{\nu}{ }^{\alpha}-\partial_{\nu} \xi_{\mu}{ }^{\alpha}\right)+i U_{B \gamma}\left(h_{\mu}{ }^{B} \xi_{\nu}{ }^{\gamma}-h_{\nu}{ }^{B} \xi_{\mu}{ }^{\gamma}\right)+i V_{\beta \gamma} \xi_{\mu}{ }^{\beta} \xi_{\nu}{ }^{\gamma}\right],
\end{aligned}
$$

which comes out to be diffeomorphism invariant. The relation (5.2) enables us to show

$$
\begin{aligned}
\delta(c, 0) \mathcal{L}= & -\partial_{\mu}\left[\epsilon ^ { \mu \nu } c ^ { B } \left\{W_{B C} h_{\nu}{ }^{C}+i U_{B \gamma} \xi_{\nu}{ }^{\gamma}-\phi_{A}\left(\frac{\partial W_{B C}}{\partial \phi_{A}} h_{\nu}{ }^{C}+i \frac{\partial U_{B \gamma}}{\partial \phi_{A}} \xi_{\nu}{ }^{\gamma}\right)\right.\right. \\
& \left.\left.-\psi_{\alpha}\left(\frac{\partial W_{B C}}{\partial \psi_{\alpha}} h_{\nu}{ }^{C}+i \frac{\partial U_{B \gamma}}{\partial \psi_{\alpha}} \xi_{\nu}{ }^{\gamma}\right)\right\}\right], \\
\delta(0, \tau) \mathcal{L}= & -\partial_{\mu}\left[i \epsilon ^ { \mu \nu } \tau ^ { \beta } \left\{U_{C \beta} h_{\nu}{ }^{C}+V_{\beta \gamma} \xi_{\nu}{ }^{\gamma}-\phi_{A}\left(\frac{\partial U_{C \beta}}{\partial \phi_{A}} h_{\nu}{ }^{C}+\frac{\partial V_{\beta \gamma}}{\partial \phi_{A}} \xi_{\nu}{ }^{\gamma}\right)\right.\right. \\
& \left.\left.-\psi_{\alpha}\left(\frac{\partial U_{C \beta}}{\partial \psi_{\alpha}} h_{\nu}{ }^{C}+\frac{\partial V_{\beta \gamma}}{\partial \psi_{\alpha}} \xi_{\nu}{ }^{\gamma}\right)\right\}\right],
\end{aligned}
$$

which confirms the invariance of our action (5.13) under the 'nonlinear' gauge transformation (5.1). The equations of motion which follow from (5.13) are given 
as follows:

$$
\begin{aligned}
& \epsilon^{\mu \nu} D_{\nu} \phi_{A}=0, \quad \epsilon^{\mu \nu} R_{\mu \nu}^{A}=0 \\
& \epsilon^{\mu \nu} D_{\nu} \psi_{\alpha}=0, \quad \epsilon^{\mu \nu} R_{\mu \nu}^{\alpha}=0
\end{aligned}
$$

which are indeed covariant due to the transformation law (5.6), (2.22).

\subsection{Generic Form of 'Dilaton' Supergravity}

As a typical example of nonlinear supergauge theory defined in previous section, we consider generic form of two-dimensional $N=1$ dilaton supergravity. $N=1$ dilaton supergravity has been defined in Ref. [25].

The superfield formalism are very useful when we construct a supersymmetric field theory. ${ }^{[23]}$ Also in this paper, we construct $N=1$ dilaton supergravity by that method. Thus we introduce superspace $\left(x^{\mu}, \theta^{\alpha}\right)$, where $\theta$ is Grassmann number and $\mu$ and $\alpha$ run over 0 and 1 .

In order to construct $N=1$ supersymmetric extension of two-dimensional dilaton gravity, we introduce a scalar superfield $\Phi$ and a superzweibein $E_{\mu}{ }^{a}$ as

$$
\begin{aligned}
& \Phi \equiv \varphi+i \bar{\theta} \chi+\frac{i}{2} \bar{\theta} \theta F, \\
& E_{\mu}{ }^{a} \equiv e_{\mu}{ }^{a}+i \bar{\theta} \gamma^{a} \zeta_{\mu}+\frac{i}{4} \bar{\theta} \theta e_{\mu}{ }^{a} A,
\end{aligned}
$$

where $\theta$ and $\bar{\theta}$ are real Grassmann numbers and $\chi$ and $\zeta_{\mu}$ are fermions. Since $\theta$ and $\bar{\theta}$ are real, $\theta$ term is not needed. Generic form of dilaton supergravity considered in this paper is the following action: ${ }^{[24][25]}$

$$
S_{G}=2 i \int d^{2} x d^{2} \theta E[\Phi S-\mathcal{Z}(\Phi)]
$$


where $\mathcal{Z}(\Phi)$ is a smooth function of $\Phi$ and

$$
\begin{aligned}
& E=e\left[1+\frac{i}{2} \bar{\theta} \gamma^{\mu} \zeta_{\mu}+\bar{\theta} \theta\left(\frac{i}{4} A+\frac{1}{8} e^{\mu \nu} \bar{\zeta}_{\mu} \gamma_{5} \zeta_{\nu}\right)\right] \\
& S=A+i \bar{\theta} \Sigma+\frac{i}{2} \bar{\theta} \theta G \\
& \Sigma=-2 e^{\mu \nu} \gamma_{5} D_{\mu} \zeta_{\nu}-\frac{1}{2} \gamma^{\mu} \zeta_{\mu} A \\
& G=-R-\frac{i}{2} \bar{\zeta}_{\mu} \gamma^{\mu} \Sigma+\frac{i}{4} e^{\mu \nu} \bar{\zeta}_{\mu} \gamma_{5} \zeta_{\nu} A-\frac{1}{2} A^{2} \\
& E_{\mu}{ }^{a}=e_{\mu}{ }^{a}+i \bar{\theta} \gamma^{a} \zeta_{\mu}+\frac{i}{4} \bar{\theta} \theta e_{\mu}{ }^{a} A \\
& E_{\mu}{ }^{\alpha}=\frac{1}{2} \zeta_{\mu}{ }^{\alpha}-\frac{1}{2}\left(\bar{\theta} \gamma_{5}\right)^{\alpha} \omega_{\mu}-\frac{1}{4}\left(\bar{\theta} \gamma_{\mu}\right)^{\alpha} A-\bar{\theta} \theta\left(\frac{3 i}{16} \zeta_{\mu}{ }^{\alpha} A+\frac{i}{4}\left(\gamma_{\mu} \Sigma\right)^{\alpha}\right) \\
& E_{\beta}{ }^{a}=i\left(\bar{\theta} \gamma^{a}\right)_{\beta}, \\
& E_{\beta}{ }^{\alpha}=\delta_{\beta}{ }^{\alpha}\left(1-\frac{i}{8} \bar{\theta} \theta A\right)
\end{aligned}
$$

Here we denote

$$
\begin{aligned}
& e^{\mu \nu}=e^{-1} \epsilon^{\mu \nu}, \\
& R=2 e^{\mu \nu} \partial_{\mu} \omega_{\nu}, \\
& D_{\mu} \zeta_{\nu}=\partial_{\mu} \zeta_{\nu}+\frac{1}{2} \omega_{\mu} \gamma_{5} \zeta_{\nu}, \\
& \omega_{\mu}=e_{\mu}{ }^{a} e^{\nu \rho} \partial_{\nu} e_{\rho a}-\frac{i}{2} \bar{\zeta}_{\mu} \gamma_{5} \gamma^{\nu} \zeta_{\nu} .
\end{aligned}
$$

and $\epsilon^{\mu \nu}$ is the Levi-Civita antisymmetric tensor, $a, b$ are tangent space indices, $\mu, \nu$ are spacetime indices and $\alpha, \beta$ are spinor indices. Moreover we set $\gamma_{5}=\gamma^{0} \gamma^{1}$.

The locally well-defined field redefinition enables us to have (5.16) from the action in Ref. [25]. The action (5.16) is invariant under the general coordinate, local-Lorentz and the following local supersymmetry transformation: 


$$
\begin{aligned}
& \delta_{\mathrm{S}} \varphi=i \bar{\epsilon} \chi \\
& \delta_{\mathrm{S}} \chi=\left[\gamma^{\mu}\left(\partial_{\mu} \varphi-\frac{i}{2} \bar{\zeta}_{\mu} \chi\right)-F\right] \epsilon \\
& \delta_{\mathrm{S}} F=i \bar{\epsilon} \gamma^{\mu}\left[-\left(\partial_{\mu}+\frac{1}{2} \omega_{\mu} \gamma_{5}\right) \chi+\frac{1}{2} \gamma^{\nu}\left(\partial_{\nu} \varphi-\frac{i}{2} \bar{\zeta}_{\nu} \chi\right) \zeta_{\mu}-\frac{1}{2} F \zeta_{\mu}\right] \\
& \delta_{\mathrm{S}} e_{\mu}{ }^{a}=i \bar{\epsilon} \gamma^{a} \zeta_{\mu} \\
& \delta_{\mathrm{S}} \zeta_{\mu}=2\left(\partial_{\mu}+\frac{1}{2} \omega_{\mu} \gamma_{5}\right) \epsilon+\frac{1}{2} \gamma_{\mu} A \epsilon \\
& \delta_{\mathrm{S}} A=i \bar{\epsilon} \Sigma
\end{aligned}
$$

where $\epsilon$ is a gauge parameter.

In the following section, we investigate a gauge-theoretical origin of the 'dilaton' superfield $\Phi$ in the action (5.16).

\subsection{Nonlinear Super-Poincaré Algebra}

In order to construct the action (5.16) as the nonlinear supergauge theory, we expand each field in terms of $\theta$ and $\bar{\theta}$, and carry out the Grassmann integration of the action (5.16). Then (5.16) becomes as follows:

$$
\begin{aligned}
S_{G}= & \int d^{2} x\left[-\frac{1}{2} \epsilon^{\mu \nu}\left\{\varphi\left(\partial_{\mu} \omega_{\nu}-\partial_{\nu} \omega_{\mu}\right)-i \bar{\chi} \gamma_{5}\left(\partial_{\mu} \zeta_{\nu}-\partial_{\nu} \zeta_{\mu}\right)-i \omega_{\mu}\left(\bar{\chi} \zeta_{\nu}\right)+\frac{1}{2} \epsilon_{a b} e_{\mu}{ }^{a} e_{\nu}{ }^{b} F A\right\}\right. \\
& \left.+\frac{1}{2} e\{i \bar{\chi} \chi \mathcal{W}(\varphi)-F \mathcal{U}(\varphi)\}+\frac{i}{4} \epsilon^{\mu \nu} \epsilon_{a b} e_{\mu}{ }^{a}\left(\bar{\zeta}_{\nu} \gamma^{b} \chi\right) \mathcal{U}(\varphi)-\frac{1}{4}\left(e A-\frac{i}{2} \epsilon^{\mu \nu} \bar{\zeta}_{\mu} \gamma_{5} \zeta_{\nu}\right) \mathcal{V}(\varphi)\right]
\end{aligned}
$$

where we have written $\mathcal{Z}(\Phi)$ as

$$
\mathcal{Z}(\Phi)=\mathcal{V}(\varphi)+i \bar{\theta} \chi \cdot \mathcal{U}(\varphi)+\frac{i}{2} \bar{\theta} \theta[-i \bar{\chi} \chi \cdot \mathcal{W}(\varphi)+F \mathcal{U}(\varphi)]
$$

$\mathcal{V}, \mathcal{U}$ and $\mathcal{W}$ being smooth functions of $\varphi$.

Next we regard the zweibein $e_{\mu}{ }^{a}$ and the spinconnection $\omega_{\mu}$ as the independent 
fields as the bosonic case ${ }^{[12]}$ by which the following term is added to the action:

$$
\begin{aligned}
S_{A} & =-\frac{1}{2} \epsilon^{\mu \nu} \int d^{2} x \phi_{a} T_{\mu \nu}^{a}, \\
T_{\mu \nu}{ }^{a} & =\partial_{\mu} e_{\nu}{ }^{a}+\omega_{\mu} \epsilon^{a b} e_{\nu b}+\frac{i}{4} \epsilon_{b}^{a}\left(\bar{\zeta}_{\mu} \gamma_{5} \gamma^{b} \zeta_{\nu}\right)-(\mu \leftrightarrow \nu) .
\end{aligned}
$$

That is, if the auxiliary field $\phi_{a}$ is integrated out, we go back to the action (5.20) together with the last equation of (5.18).

Moreover since $A$ and $F$ are auxiliary fields, they are eliminated from the action (5.20) by using the equations of motion of $A$ and $F$ :

$$
\begin{aligned}
A & =\mathcal{U}(\varphi), \\
F & =\frac{1}{2} \mathcal{V}(\varphi) .
\end{aligned}
$$

The final expression for the action is as follows:

$$
\begin{aligned}
S_{P}= & \int d^{2} x \mathcal{L}_{P} \\
\mathcal{L}_{P}= & -\frac{1}{2} \epsilon^{\mu \nu}\left[\varphi\left(\partial_{\mu} \omega_{\nu}-\partial_{\nu} \omega_{\mu}\right)-i \bar{\chi} \gamma_{5}\left(\partial_{\mu} \zeta_{\nu}-\partial_{\nu} \zeta_{\mu}\right)+\phi_{a}\left(\partial_{\mu} e_{\nu}{ }^{a}-\partial_{\nu} e_{\mu}{ }^{a}\right)\right. \\
& -\left\{\frac{1}{4} \mathcal{U}(\varphi) \mathcal{V}(\varphi)-i \bar{\chi} \chi \mathcal{W}(\varphi)\right\} \epsilon_{a b} e_{\mu}{ }^{a} e_{\nu}{ }^{b}+2 \omega_{\mu}\left(\phi_{a} \epsilon^{a b}\right) e_{\nu b} \\
& -i \bar{\chi} \omega_{\mu} \zeta_{\nu}+\frac{i}{2} \bar{\chi} \gamma^{a} \epsilon_{a b} \mathcal{U} e_{\mu}{ }^{b} \zeta_{\nu} \\
& \left.-\frac{i}{2} \bar{\zeta}_{\mu}\left\{\frac{1}{2} \gamma_{5} \mathcal{V}(\varphi)-\phi_{a} \epsilon^{a}{ }_{b} \gamma_{5} \gamma^{b}\right\} \zeta_{\nu}\right] .
\end{aligned}
$$

This action (5.22) is classically equivalent to (5.16). Comparing (5.22) with (5.13), we find the base nonlinear superalgebra has the following commutation relation:

$$
\begin{aligned}
& {\left[J, P_{a}\right]=\epsilon_{a b} \eta^{b c} P_{c}} \\
& {\left[P_{a}, P_{b}\right]=-\frac{1}{4} \epsilon_{a b} \mathcal{U}(J) \mathcal{V}(J)-\frac{i}{2} \epsilon_{a b}\left(Q \gamma^{0} Q\right) \mathcal{W}(J),} \\
& {\left[J, Q_{\alpha}\right]=\frac{1}{2}\left(Q \gamma_{5}\right)_{\alpha}} \\
& {\left[P_{a}, Q_{\beta}\right]=\frac{1}{4}\left(Q \gamma^{0} \gamma^{b} \gamma^{1} \epsilon_{b a}\right)_{\alpha} \mathcal{U}(J),} \\
& \left\{Q_{\alpha}, Q_{\beta}\right\}=\frac{i}{2}\left(\gamma^{a} \gamma^{0}\right)_{\alpha \beta} P_{a}+\frac{i}{4}\left(\gamma^{1}\right)_{\alpha \beta} \mathcal{V}(J) .
\end{aligned}
$$


and the other commutation relations vanish. Here we set $\left\{T_{A}\right\}=\left\{P_{a}, J\right\}$, that is, $T_{0}=P_{0}, T_{1}=P_{1}$, and $T_{2}=J$, and we also set the vector fields $h_{\mu}^{A}=\left(e_{\mu}{ }^{a}, \omega_{\mu}\right)$, $\xi_{\mu}^{\alpha}=\left(\gamma^{1} \zeta_{\mu}\right)^{\alpha}$ and the scalar field $\phi_{A}=\left(\phi_{a}, \varphi\right), \psi_{\alpha}=\chi_{\alpha}^{\dagger} ; \eta^{c d}$ is the twodimensional Minkowski metric.

Note that the choice $\mathcal{W}(J)=\mathcal{V}(J)=\mathcal{U}(J)=0$ corresponds to the original super-Poincaré algebra.

Then the structure functions defined in (2.23) for the above algebra are given by

$$
\begin{aligned}
W_{2 a} & =-W_{a 2}=\epsilon_{a}^{b} \phi_{b}, \quad W_{22}=0, \\
W_{a b} & =-\frac{1}{4} \epsilon_{a b} \mathcal{U}(\varphi) \mathcal{V}(\varphi)+\frac{i}{2} \epsilon_{a b} \bar{\chi} \chi \mathcal{W}(\varphi), \\
U_{2 \beta} & =-\frac{1}{2} \bar{\chi}_{\beta}, \\
U_{a \beta} & =\frac{1}{4}\left(\bar{\chi} \gamma^{b} \gamma^{1}\right)_{\beta} \epsilon_{b a} \mathcal{U}(\varphi), \\
V_{\alpha \beta} & =-\frac{1}{4}\left(\gamma^{0} \gamma_{5}\right)_{\alpha \beta} \mathcal{V}(\varphi)-\frac{1}{2} \phi_{a}\left(\gamma^{a} \gamma^{0}\right)_{\alpha \beta} .
\end{aligned}
$$

The gauge transformation law (5.1) now reads

$$
\begin{aligned}
\delta \omega_{\mu}= & \partial_{\mu} t+\epsilon_{b c} c^{b} e_{\mu}{ }^{c} \frac{\partial}{\partial \varphi}\left[\frac{1}{4} \mathcal{U}(\varphi) \mathcal{V}(\varphi)-\frac{i}{2} \bar{\chi} \chi \mathcal{W}(\varphi)\right] \\
& +\frac{i}{4} \epsilon_{b c} c^{b}\left(\bar{\chi} \gamma^{c} \zeta_{\mu}\right) \frac{\partial \mathcal{U}}{\partial \varphi}+\frac{i}{4}\left(\bar{\chi} \gamma^{b} \tau\right) \epsilon_{b c} e_{\mu}{ }^{c} \frac{\partial \mathcal{U}}{\partial \varphi}+\frac{i}{4}\left(\bar{\tau} \gamma_{5} \zeta_{\mu}\right) \frac{\partial \mathcal{V}}{\partial \varphi} \\
\delta e_{\mu}{ }^{a}= & -t \epsilon^{a b} e_{\mu b}+\partial_{\mu} c^{a}+\omega_{\mu} \epsilon^{a b} c_{b}-\frac{i}{2} \epsilon^{a b}\left(\bar{\tau} \gamma_{5} \gamma_{b} \zeta_{\mu}\right), \\
\delta \zeta_{\mu}{ }^{\alpha}= & -\frac{1}{2} t\left(\gamma_{5} \zeta_{\mu}\right)^{\alpha}+\epsilon_{b c} c^{b} e_{\mu}{ }^{c}\left(\gamma_{5} \chi\right)^{\alpha} \mathcal{W}(\varphi)-\frac{1}{4} \epsilon_{b c} c^{b}\left(\gamma_{5} \gamma^{c} \zeta_{\mu}\right)^{\alpha} \mathcal{U}(\varphi) \\
& +\partial_{\mu} \tau^{\alpha}-\frac{1}{4}\left(\gamma_{5} \gamma^{b} \tau\right)^{\alpha} \epsilon_{b c} e_{\mu}{ }^{c} \mathcal{U}(\varphi), \\
\delta \varphi= & \epsilon^{a b} c_{a} \phi_{b}+\frac{i}{2} \bar{\chi} \tau, \\
\delta \phi_{a}= & -t \epsilon_{a b} \phi^{b}+\epsilon_{a b} c^{b}\left[\frac{1}{4} \mathcal{U}(\varphi) \mathcal{V}(\varphi)-\frac{i}{2} \bar{\chi} \chi \mathcal{W}(\varphi)\right] \\
\delta \chi^{\alpha}= & -\frac{1}{2} t\left(\gamma_{5} \chi\right)^{\alpha}+\frac{1}{4}\left(\gamma_{5} \gamma^{a} \chi\right)^{\alpha} \epsilon_{a b} c^{b} \mathcal{U}(\varphi)-\frac{1}{4} \mathcal{V}(\varphi) \tau^{\alpha}+\frac{1}{2} \phi^{a} \epsilon_{a b}\left(\gamma^{b} \tau\right)^{\alpha},
\end{aligned}
$$

where we have put $c^{A}=\left(c^{a}, t\right)$. 
The equations of motion which follow from (5.22) are given by

$$
\begin{aligned}
& \partial_{\mu} \varphi+\phi_{a} \epsilon^{a b} e_{\mu b}-\frac{i}{2}\left(\bar{\chi} \zeta_{\mu}\right)=0, \\
& \partial_{\mu} \phi_{a}+\omega_{\mu} \epsilon_{a b} \phi^{b}+\epsilon_{a b} e_{\mu}{ }^{b}\left(-\frac{1}{4} \mathcal{U}(\varphi) \mathcal{V}(\varphi)+\frac{i}{2} \bar{\chi} \chi \mathcal{W}(\varphi)\right)-\frac{i}{4} \epsilon_{a b}\left(\bar{\chi} \gamma^{b} \zeta_{\nu}\right) \mathcal{U}=0, \\
& \partial_{\mu} \bar{\chi}-\frac{1}{2} \bar{\chi} \gamma_{5} \omega_{\mu}+\frac{1}{4} \bar{\chi} \gamma_{5} \gamma^{a} \epsilon_{a b} e_{\mu}{ }^{b} \mathcal{U}-\frac{1}{2} \bar{\zeta}_{\mu}\left(\frac{1}{2} \mathcal{V}-\gamma^{a} \epsilon_{a b} \phi^{b}\right)=0, \\
& \frac{1}{2} \epsilon^{\mu \nu}\left[\partial_{\mu} \omega_{\nu}-\partial_{\nu} \omega_{\mu}+\epsilon_{b c} e_{\mu}{ }^{b} e_{\nu}{ }^{c} \frac{\partial}{\partial \varphi}\left\{\frac{1}{4}(\mathcal{U} \mathcal{V})+\frac{i}{2} \bar{\chi} \chi \mathcal{W}\right\}-\frac{i}{2} \epsilon_{b c} e_{\mu}{ }^{b}\left(\bar{\chi} \gamma^{c} \zeta_{\nu}\right) \frac{\partial \mathcal{U}}{\partial \varphi}-\frac{i}{4} \frac{\partial \mathcal{V}}{\partial \varphi}\left(\bar{\zeta}_{\mu} \gamma_{5} \zeta_{\nu}\right)\right]=0, \\
& \frac{1}{2} \epsilon^{\mu \nu}\left[\partial_{\mu} \zeta_{\nu}-\partial_{\nu} \zeta_{\mu}-\epsilon_{b c} e_{\mu}{ }^{b} e_{\nu}{ }^{c} \mathcal{W} \gamma_{5} \chi+\omega_{\mu} \gamma_{5} \zeta_{\nu}+\frac{1}{2} \epsilon_{b c} e_{\mu}{ }^{b} \mathcal{U} \gamma_{5} \gamma^{c} \zeta_{\nu}\right]=0, \\
& \frac{1}{2} \epsilon^{\mu \nu} T_{\mu \nu}{ }^{a}=0 .
\end{aligned}
$$

They of course correspond to (5.15).

Note that the gravitation theory (5.20) is obtained if one adds (5.21) to the Lagrangian (5.22) and integrates out the fields $\phi_{a}$ and $\omega_{\mu}$ in the Lagrangian (5.22) under the condition $e \neq 0$. The reformulation (5.22) reveals that the theory (5.16) possesses hidden gauge symmetry (5.25) of the Yang-Mills type. This gauge symmetry includes diffeomorphism, as is the case for the Yang-Mills-like formulation of generic form of dilaton gravity. ${ }^{[12]}$

\section{Conclusion}

We have made a systematic construction of the nonlinear gauge theory (2.31) with the gauge transformation (2.16) based on a general nonlinear Lie algebra. A new category of the gauge theory has been constructed as an extension of the gauge theory based on the usual Lie algebra. We have carried out the BRS quantization of nonlinear gauge theory. Since the gauge is open, we have had to modify usual quantization method. The BRS charge has been explicitly constructed without anomaly in the cylindrical spacetime $S^{1} \times \boldsymbol{R}^{1}$ in the temporal gauge. 
The algebraic structure of the above nonlinear gauge theory seems to be clear by construction. However the field $\Phi_{A}$ is not a representation of nonlinear Lie algebra; it is different from the coadjoint field of usual Lie algebra. The relation of $\Phi_{A}$ with the algebra deserves further investigation.

The clarification of its geometric structure is desirable in view of the rich structure present in the usual nonabelian gauge theory. However, the gauge algebra (2.17) is open, which implies non-existence of Lie-group-like object corresponding to the nonlinear Lie algebra. That might cause difficulty in the geometrical investigation of the nonlinear gauge theory.

In the chapters 3 and 4 , we have considered typical examples which have nonlinear gauge symmetry defined in previous chapter. We have considered Lorentzcovariant quadratic extension (4.6) of the Poincaré algebra in two dimensions in chapter 3. The nonlinear gauge theory based on it has turned out to be the Yang-Mills-like formulation of $R^{2}$ gravity with dynamical torsion. Namely, the "deformation" we observed in Ref.[10] has been shown to be Lorentz-covariant quadratically nonlinear extension described above.

We have also considered general Lorentz-covariant nonlinear extension (4.6) of the

Poincaré algebra in two dimensions in chapter 4. The nonlinear gauge theory based on its algebra has turned out to be the generic form of 'dilaton' gravity, which clarifies a gauge-theoretical origin of the non-geometric scalar field in twodimensional gravitation theory. We note that this theory is a generalization of Yang-Mills-like formulation of the Jackiw-Teitelboim's model ${ }^{[9]}$ and the dilaton gravity, ${ }^{[26]}$ because the latter corresponds to a particular choice $\mathcal{W}(\varphi)=$ constant.

In chapter 5, we have made a construction of the nonlinear supergauge theory (5.13) with the gauge transformation (5.1) based on a general nonlinear Lie superalgebra.

We have also found that the generic form of 'dilaton' supergravity turns out to be the nonlinear supergauge theory based on the Lorentz-covariant nonlinear ex- 
tension (5.23) of the super-Poincaré algebra in two dimensions. The reformulation (5.22) reveals that the theory (5.16) possesses hidden gauge symmetry (5.25) of the Yang-Mills type. We also can interpret two formulations of dilaton supergravity that the super structure of the base space in dilaton supergravity is transmuted into the super structure of target space in a nonlinear super-Poincaré algebra. This theory is a generalization of Yang-Mills-like formulation of the dilaton supergravity. ${ }^{[27]}$

We expect that construction of the nonlinear gauge theory and its application to two-dimensional gravity in this paper has offered a new method to analyze quantum field theory. The analysis of this theory at the quantum level is still insufficient. It might be interesting to compare the theory (5.16) of the Utiyama type and the one (5.22) of the Yang-Mills type in the aspects of their quantization.

\section{ACKNOWLEDGEMENTS}

The author would like to thank N. Nakanishi for valuable comments and careful reading of the manuscript. He thanks K. -I. Izawa and for valuable discussion as his collaborator. He also thanks I. Ojima and M. Abe for valuable comments and discussions. 


\section{REFERENCES}

1. for example, J. Wess and J. Bagger, Supersymmetry and Supergravity (Princeton University Press, 1983).

2. D. Bernard, Suppl. Progr. Theor. Phys. 102 (1990) 49;

I. Ya. Aref'eva and I. V. Volovich, Mod. Phys. Lett. A6 (1991) 893;

M. Hirayama, Prog. Theor. Phys. 88 (1992) 111.

3. for review, P. Bouwknegt and K. Schoutens, Phys. Rep. 223 (1993) 183.

4. R. Utiyama, Phys. Rev. 101 (1956) 1597

5. C. N. Yang and R. I. Mills, Phys. Rev. 96 (1954) 191.

6. K. Schoutens, A. Sevrin and P. van Nieuwenhuizen, Commun. Math. Phys. 124 (1989) 87; Int. J. Mod. Phys. A6 (1991) 2891; Phys. Lett. B255 (1991) 549 .

7. E. Witten, Nucl. Phys. B311 (1988/89) 46; B323 (1989) 113.

8. C. Teitelboim, Phys. Lett. B126 (1983) 41; in Quantum Theory of Gravity, ed. S.M. Christensen (Adam Hilger, 1984);

R. Jackiw, in Quantum Theory of Gravity, ed. S.M. Christensen (Adam Hilger, 1984); Nucl. Phys. B252 (1985) 343;

M. Henneaux, Phys. Rev. Lett. 54 (1985) 959;

See also M. Abe and N. Nakanishi, Int. J. Mod. Phys. A6 (1991) 3955.

9. T. Fukuyama and K. Kamimura, Phys. Lett. B160 (1985) 259;

K. Isler and C.A. Trugenberger, Phys. Rev. Lett. 63 (1989) 834;

A.H. Chamseddine and D. Wyler, Phys. Lett. B228 (1989) 75; Nucl. Phys. B340 (1990) 595;

H. Terao,, Nucl. Phys. B395 (1993) 623;

See also D. Cangemi and R. Jackiw, Phys. Rev. Lett. 69 (1992) 233.

10. N. Ikeda and Izawa K.-I., Prog. Theor. Phys. 89 (1993) 223.

11. N. Ikeda and Izawa K.-I., Prog. Theor. Phys. 89 (1993) 1077. 
12. N. Ikeda and Izawa K.-I., Prog. Theor. Phys. 90 (1993) 237.

13. N. Ikeda Int. J. Mod. Phys. A, to be published.

14. A.B. Zamolodchikov, Theor. Math. Phys. 65 (1986) 1205.

15. V. G. Drinfel'd, in Proceedings of International Congress of Mathematicians (Berkeley, CA, USA, 1986) 793;

M. Jimbo, Lett. Math. Phys. 10 (1985) 63.

16. M. Roček, Phys. Lett. B255 (1991) 554;

T. Tjin, Phys. Lett. B292 (1992) 60;

J. de Boer and T. Tjin, preprint THU-92-32.

17. I.A. Batalin and G.A. Vilkovisky, Phys. Lett. B102 (1981) 27; Phys. Rev. D28 (1983) 2567; J. Math. Phys. 26 (1985) 172.

18. T. Kugo and I. Ojima, Phys. Lett. B73 (1978) 459; Suppl. Prog. Theor. Phys. 66 (1979) 1;

M. Kato and K. Ogawa, Nucl. Phys. B212 (1983) 443;

N. Nakanishi and I. Ojima, Covariant Operator Formalism of Gauge Theories and Quantum Gravity (World Scientific, 1990).

19. M.O. Katanaev and I.V. Volovich, JETP Lett. 43 (1986) 267; Phys. Lett. B175 (1986) 413; Ann. of Phys. 197 (1990) 1;

M.O. Katanaev, Theor. Math. Phys. 80 (1989) 838; Sov. Phys. Dokl. 34 (1989) 982; J. Math. Phys. 31 (1990) 882; 32 (1991) 2483;

K.G. Akdeniz, A. Kizilersü, and E. Rizaoğlu, Phys. Lett. B215 (1988) 81;

K.G. Akdeniz, Ö.F. Dayi, and A. Kizilersü, Mod. Phys. Lett. A7 (1992) 1757 ;

W. Kummer and D.J. Schwarz, Phys. Rev. D45 (1992) 3628; Nucl. Phys. B382 (1992) 171;

H. Grosse, W. Kummer, P. Prešnajder, and D.J. Schwarz, J. Math. Phys. 33 (1992) 3892;

T. Strobl,, Int. J. Mod. Phys.A8 (1993) 1383; 
P. Schaller and T. Strobl, preprint TUW-92-13, TUW-93-08;

H. Haider and W. Kummer, preprint TUW-92-15

20. T. Yoneya, Phys. Lett. B149 (1984) 111;

A.M. Polyakov, Mod. Phys. Lett. A2 (1987) 893.

21. T. Strobl, Int. J. Mod. Phys.A8 (1993) 1383

22. J.G. Russo and A.A. Tseytlin, Nucl. Phys. B382 (1992) 259.

23. P. Fayet and S. Ferrara, Phys. Rep. 32C (1977) 249

24. P. S. Howe, J. phys. A:Math. Gen. 12 (1979) 393.

25. S. Nojiri and I. Oda, Mod. Phys. Lett. A8 (1993) 53, Y. Park and A. Strominger, Phys. Rev. D47 (1993) 1569, A. Bilal, Phys. Rev. D48 (1993) 1665.

26. H. Verlinde, in The Sixth Marcel Grossmann Meeting on General Relativity, ed. M. Sato (World Scientific, Singapore, 1992);

D. Cangemi and R. Jackiw, Phys. Rev. Lett. 69 (1992) 233.

27. V. O. Rivelles preprint IFUSP-P-1025 (1992). 